\title{
Accelerating XOR-based Erasure Coding using Program Optimization Techniques
}

\author{
Yuya Uezato \\ Dwango, Co., Ltd. \\ Tokyo, Japan \\ yuuya_uezato@dwango.co.jp
}

\begin{abstract}
Erasure coding (EC) affords data redundancy for large-scale systems. XOR-based EC is an easy-to-implement method for optimizing EC. This paper addresses a significant performance gap between the state-of-the-art XOR-based EC approach ( $\sim 4.9 \mathrm{~GB} / \mathrm{s}$ coding throughput) and Intel's high-performance EC library based on another approach $(\sim 6.7 \mathrm{~GB} / \mathrm{s})$. We propose a novel approach based on our observation that XOR-based EC virtually generates programs of a Domain Specific Language for XORing byte arrays. We formalize such programs as straight-line programs (SLPs) of compiler construction and optimize SLPs using various optimization techniques. Our optimization flow is three-fold: 1) reducing operations using grammar compression algorithms; 2) reducing memory accesses using deforestation, a functional program optimization method; and 3) reducing cache misses using the (red-blue) pebble game of program analysis. We provide an experimental library, which outperforms Intel's library with a $\sim 8.92 \mathrm{~GB} / \mathrm{s}$ throughput.
\end{abstract}

\section{INTRODUCTION}

Assuring data redundancy is the most critical task for large-scale systems such as distributed storage. Replication-distributing the replicas of data-is the simplest solution. Erasure coding (EC) has attracted significant attention thanks to its space efficiency [101] For example, the famous distributed system HDFS (Hadoop Distributed File System) [8] offers the codec RS(10, 4), Reed-Solomon EC [84] with 10 data blocks and 4 parity blocks. On RS(10, 4), we can store 10-times more objects than through replication; however, we cannot recover data if five nodes are down. Another distributed system, Ceph [24], offers $\mathbf{R S}(n, p)$ for any $n$ and $p$. On Linux, we can use RAID-6, a codec similar to $\mathbf{R S}(n, 2)[11,82]$. Using EC instead of the replication degrades the system performance since the encoding and decoding of EC are heavy computation and are required for each storing to and loading from a system. It is often stated that EC is suitable only for archiving cold (rarely accessed) data $[29,49,93]$.

We clarify the pros and cons of EC by observing how RS works. To encode data using matrix multiplication (hereafter we use the acronym MM), RS adopts matrices over $\mathbb{F}_{2^{8}}$, the finite field with $2^{8}=256$ elements. Since each element of $\mathbb{F}_{2^{8}}$ is coded by one byte ( 8 bits), we can identify an $N$-bytes data as an $N$-array of $\mathbb{F}_{2^{8}}$. RS $(n, p)$ encodes an $N$-byte data $D$ using an $(n+p) \times n$ Vandermonde matrix $\mathcal{V} \in \mathbb{F}_{2^{8}}^{(n+p) \times n}$, which is key for decoding as we will see below, as

This is the author (and non-final) version of the same title paper that accepted by SC'21 https://sc21.supercomputing.org/. The final version will be published by ACM in the DOI https://doi.org/10.1145/3458817.3476204. The appendix contains a proof of Theorem 1 omitted from the conference version due to page limitation. follows:

$$
\begin{gathered}
n \\
+ \\
p
\end{gathered} \mathbb{F}_{2^{8}}\left(\begin{array}{c}
\vec{d}_{1} \\
\vdots \\
\vec{d}_{n}
\end{array}\right)=\left(\begin{array}{c}
\vec{b}_{1} \\
\vdots \\
\vec{b}_{n} \\
\vdots \\
\vec{b}_{n+p}
\end{array}\right) \begin{aligned}
& \text { where } \\
& \cdot \mathbb{F}_{2^{8}} \text { is the MM over } \mathbb{F}_{2^{8}} ; \\
& \vec{d}_{i} \text { is } i \text {-th } \frac{N}{n} \text {-bytes block of } D ; \\
& \vec{b}_{j} \text { is an } \frac{N}{n} \text {-bytes coded block. }
\end{aligned}
$$

We store an encoded block $\vec{b}_{i}$ to a node $n_{i}$ of a system with $n+p$ nodes. For decoding, we gather $n$-blocks $B=\left(\vec{b}_{i_{1}} \vec{b}_{i_{2}} \ldots \vec{b}_{i_{n}}\right)^{T}$ from alive nodes. The $(n \times n)$-submatrix $\mathcal{M}$ of $\mathcal{V}$ obtained by extracting row-vectors at $\left\{i_{1}, i_{2}, \ldots, i_{n}\right\}$ satisfies $B=\mathcal{M} \cdot \mathbb{F}_{2^{8}} D$. Since any square submatrix of Vandermonde matrices is invertible [65, 73, 94], the inverse $\mathcal{M}^{-1}$ of $\mathcal{M}$ recovers $D$ as $\mathcal{M}^{-1} \cdot \mathbb{F}_{2^{8}} B=D$.

Now, the advantage of RS space efficiency emerges as the size of the encoded blocks. For example, on $\mathbf{R S}(10,4)$, nodes of a system require $\frac{N}{10}$-bytes of space for an $N$-bytes data. On the other hand, the disadvantage slowness results from MM over finite fields. Multiplying $n \times n$ matrices requires $\sim O\left(n^{2.37287}\right)$ field operations even when using the latest result [5, 62]. Moreover, finite field multiplication $\times_{\mathbb{F}_{k}}$ is computationally expensive, and its optimization is an active research area $[50,57,59,66,83]$.

There are two primary acceleration methods of RS

(1) Tightly coupling sophisticated optimization methods for MM and finite field multiplication. Intel provides an EC library, ISA-L (Intelligent Storage Acceleration Library), based on this approach [52]. ISA-L is exceptionally optimized for MM over $\mathbb{F}_{2^{8}}$ and offers different assembly codes for each platform to maximize SIMD instruction performance $[7,10,56]$. Intel reported ISA-L scored about $6.0 \mathrm{~GB} / \mathbf{s}$ encoding throughput for $\mathbf{R S}(10,4)$ in [54]. In our evaluation at $\$ 7$, ISA-L scores for $6.7 \mathrm{~GB} / \mathrm{s}$.

(2) XOR-based EC $[13,74,103]$ converts $M M$ over $\mathbb{F}_{2^{8}}$ to $M M$ over $\mathbb{F}_{2}$ where $\mathbb{F}_{2}$ is the finite field of the bits $\{0,1\}$. This approach depends on the following two properties:

(i) There is an isomorphism $\mathfrak{B}: \mathbb{F}_{2^{8}} \cong \mathbb{F}_{2}^{8 \times 1}$ between bytes and 8-bits column vectors;

(ii) There is a function $\widetilde{\sim}: \mathbb{F}_{2^{8}} \rightarrow \mathbb{F}_{2}^{8 \times 8}$ from bytes to $8 \times 8$ matrices over $\mathbb{F}_{2}$ such that: $\forall x, y \in \mathbb{F}_{2^{8}}, x \times_{\mathbb{F}_{28}} y=\mathfrak{B}^{-1}\left(\tilde{x} \cdot \mathbb{F}_{2} \mathfrak{B}(y)\right)$.

We can calculate the above $\mathcal{V} \cdot \mathbb{F}_{2^{8}} D$ without the finite field

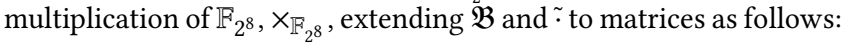

$$
\mathcal{V} \cdot \mathbb{F}_{2^{8}} D=\mathfrak{B}^{-1}\left(\tilde{\mathcal{V}} \cdot \mathbb{F}_{2} \mathfrak{B}(D)\right) .
$$

Since the addition (resp. multiplication) of $\mathbb{F}_{2}$ is the bit XOR $x \oplus y$ (resp. bit AND), MM over $\mathbb{F}_{2}$ is just array XORs, as presented below:

$$
\left(\begin{array}{c}
1100000 \\
0011110 \\
0011101
\end{array}\right) \cdot \mathbb{F}_{2}\left(\begin{array}{c}
\vec{d}_{1} \\
\vdots \\
\vec{d}_{7}
\end{array}\right)=\left(\begin{array}{c}
\vec{d}_{1} \oplus \vec{d}_{2} \\
\vec{d}_{3} \oplus \vec{d}_{4} \oplus \vec{d}_{5} \oplus \vec{d}_{6} \\
\vec{d}_{3} \oplus \vec{d}_{4} \oplus \vec{d}_{5} \oplus \vec{d}_{7}
\end{array}\right) .
$$


MM over $\mathbb{F}_{2}$ is easy-to-implement. Thanks to its implementability, this method was proposed in VLSI to realize finite field arithmetic on a small circuit [74]. This method is currently receiving attention since array XORs $\vec{d}_{i} \oplus \vec{d}_{j}$ are quickly executed via recent SIMD instructions [83]. In exchange for the ease of implementation, the obtained coding matrix $\tilde{V} \in \mathbb{F}_{2}^{8 a \times 8 b}$ is much larger than the original coding matrix $\mathcal{V} \in \mathbb{F}_{2^{8}}^{a \times b}$; thus, $\tilde{V}$ needs more (but simple) operations of $\mathbb{F}_{2}$ than those of $\mathbb{F}_{2^{8}}$ in $\mathcal{V}$.

Recently, Zhou and Tian published an invaluable study [103] that synthesized several acceleration methods for executing array XORs. It is the state-of-the-art study based on XOR-based EC; however, it scored 4.9 GB/s for $\mathbf{R S}(10,4)$ encoding.

Now, we have a question: "Is XOR-based EC essentially slower than the former approach in exchange for the ease of implementation?". The answer is "No". We provide a streamlined method to optimize XOR-based EC by employing various program optimization techniques. We also implement and provide an experimental EC library outperforming ISA-L.

\section{OUR APPROACH AND CONTRIBUTION}

We identify the $\mathrm{MM}$ over $\mathbb{F}_{2},{ }^{\cdot} \mathbb{F}_{2}$, as straight-line programs (SLPs), a classical compiler theory tool $[2,3]$, as follows:

$$
\left(\begin{array}{c}
1100000 \\
0011110 \\
0011101
\end{array}\right) \cdot \mathbb{F}_{2}\left(\begin{array}{c}
\vec{a} \\
\vec{b} \\
\vdots \\
\vec{g}
\end{array}\right) \Leftrightarrow P: \begin{aligned}
& v_{1} \leftarrow a \oplus b \\
& v_{2} \leftarrow c \oplus d \oplus e \oplus f \\
& v_{3} \leftarrow c \oplus d \oplus e \oplus g \\
& \operatorname{ret}\left(v_{1}, v_{2}, v_{3}\right)
\end{aligned}
$$

where $a, b, \ldots, g$ are constants meaning input arrays, and $v_{1}, v_{2}, v_{3}$ are variables meaning arrays allocated at runtime. SLPs are programs with a single binary operator without branchings, loops, and functions as above.

Replacing the MM over $\mathbb{F}_{2}$ by SLPs is a simple but key idea for importing various optimization methods from theory of programming. This is the crucial difference between our study and that of Zhou and Tian [103], where they treated topics directly on matrices of $\mathbb{F}_{2}$ and introduced ad-hoc constructions, without sophisticated results of program optimization.

Our Goal. The goal of this paper is to provide an efficient EC library importing various programmer-friendly optimization methods from theory of programming. Technically, we implement our optimizer as a translator, which converts an SLP to a more efficient one. When encoding and decoding data, we run optimized SLPs line-by-line in our host language in the interpreter style.

\subsection{Idea and Contribution in Our Optimizer}

We optimize SLPs via compressing, fusing, and scheduling. Let us see the idea of each step by optimizing the above $P$ as follows:

$$
\begin{aligned}
& \lambda \leftarrow c \oplus d \oplus e ; \quad \lambda \leftarrow \bigoplus(c, d, e) ; \quad v_{1} \leftarrow a \oplus b ; \\
& v_{1} \leftarrow a \oplus b ; \quad v_{1} \leftarrow a \oplus b ; \quad \lambda \leftarrow \bigoplus(c, d, e) ; \\
& P \Rightarrow v_{2} \leftarrow \lambda \oplus f ; \quad \Leftrightarrow \quad v_{2} \leftarrow \lambda \oplus f ; \quad \Leftrightarrow \quad v_{2} \leftarrow \lambda \oplus f ; \\
& v_{3} \leftarrow \lambda \oplus g ; \text { fuse } v_{3} \leftarrow \lambda \oplus g ; \text { sched. } \quad \lambda \leftarrow \lambda \oplus g \text {; } \\
& \operatorname{ret}\left(v_{1}, v_{2}, v_{3}\right) \quad \operatorname{ret}\left(v_{1}, v_{2}, v_{3}\right) \quad \operatorname{ret}\left(v_{1}, v_{2}, \lambda\right)
\end{aligned}
$$

Compressing. We use the compression algorithm REPAIR [67], which is used to compress context-free grammars (CFGs) in grammar compression. We can immediately adapt it by ignoring $\oplus$ of
SLPs, and by identifying constants (resp. variables) of SLPs as terminals (resp. nonterminals) of CFGs.

RePAIR compresses a program (or CFG) by extracting its hidden repetition structures. For $P$, REPAIR extracts the repeatedly appearing subterm $c \oplus d \oplus e$ and replaces it with a new variable $\lambda$. It reduces the seven XORs to five and speeds up $\frac{5}{7} \sim 30 \%$.

We extend RePAIR to XorRePAIR by adding the XOR-cancellation property $(x \oplus x \oplus y=y)$, not considered in grammar compression.

Fusing. To reduce memory access, we employ a technique called deforestation in functional program optimization [100]. Deforestation eliminates intermediate data via fusing functions. Although it has a deep background theory, we can easily adapt it thanks to the simplicity of SLP (one operator and no functions).

In our example, $c \oplus d \oplus e$ invokes six memory accesses because $c \oplus d$ invokes three-loading $c, d$ and storing the result to an intermediate array $I_{c, d}$, and the remaining $I_{c, d} \oplus e$ also invokes three. By fusing the two XORs to $\bigoplus(c, d, e)$, we eliminate (deforest) the intermediate array $I_{c, d}$. The fused XOR only invokes four memory accesses; loading $c, d$, and $e$, and storing the result array.

Scheduling. To reduce cache misses, we revisit the well-known (but vague) maxim for cache optimization increasing the locality of data access. It appears in our example to reorder $\lambda$ and $v_{1}$ to adjust the generation site of $\lambda$ to the use sites, $\lambda \oplus f$ and $\lambda \oplus g$. Furthermore, we reuse $\lambda$ without allocating and accessing $v_{3}$.

The maxim for cache optimization is too vague to automatically optimize SLPs and incorporate it into our optimizer. Thus, in §6, we introduce measures for cache efficiency and concretize our optimization problem as reducing the measures of a given SLP. To

\begin{tabular}{|c|c|c|c|c|c|c|}
\hline $\begin{array}{c}\text { Base: } \\
4.03 \mathrm{~GB} / \mathrm{s}\end{array}$ & $\stackrel{\text { Comp. }}{\rightarrow}$ & $\begin{array}{c}\text { In } \S 4: \\
4.36 \mathrm{~GB} / \mathrm{s}\end{array}$ & $\stackrel{\text { Fuse }}{\longrightarrow}$ & $\begin{array}{c}\text { In } \S 5: \\
7.50 \mathrm{~GB} / \mathrm{s}\end{array}$ & $\stackrel{\text { Sched. }}{\longrightarrow}$ & $\begin{array}{c}\text { In } \S 6: \\
8.92 \mathrm{~GB} / \mathrm{s}\end{array}$ \\
\hline
\end{tabular}
optimize SLPs, we employ the (red-blue) pebble game of program analysis [47, 91]. The game is a simple abstract model of computation with fast and slow devices. In our setting, the fast and slow devices are cache and main memory, respectively.

Performance. Each step improves coding performance as follows.

where Base runs unoptimized SLPs that are obtained from matrices, such as the above $P$. Interestingly, although (Xor)RePAIR reduces about $60 \%$ XORs on average (as we will see in $§ 7$ ), the summary says the compressing effect is small. This is because (XOR)RePAIR generates cache-poor compressed SLPs, and this observation will be substantiated by the cache analysis using our pebble game. The sole application of (XOR)REPAIR is not good as the theoretical improvements suggest; however, compressing achieves excellent performance in combination with memory and cache optimization.

\section{RELATED WORK}

Zhou and Tian earnestly studied and evaluated various acceleration techniques for XOR-based EC $[48,72,82]$ in [103]. Their study comprises two stages: (i) reducing XORs of bitmatrices (= matrices over $\mathbb{F}_{2}$ ) [48, 82]; and (ii) reordering XORs for cache optimization [72]. We emphasize that the previous works [48, 72, 82]-and thus, Zhou and Tian-never considered SLPs, deforestation, and pebble games. 
The lack of considering SLP makes a problem in each stage. First, the XOR reducing heuristics of $[48,82]$ run on graphs, which are obtained in an ad-hoc manner from bitmatrices. This leads to a lack of considering the XOR-cancellation (unlike our XorRePAIR) and limited performance. Indeed, Zhou and Tian reported the average reducing ratio (the smaller the better) $\frac{\# X O R \text { of reduced }}{\# X O R \text { of original }} \approx 65 \%$. Their ratio is larger than ours $-42.1 \%$ of REPAIR and $40.8 \%$ of XORREPAIR, as we will see in $§ 7$. Next, the cache optimization heuristics of [72], which reorders XORs locally without considering pebble games, is not quite effective, $\frac{\text { throughput of optimized }}{\text { throughput of original }} \approx 101 \%$ in [103]. In contrast, our heuristics for the scheduling problem are effective, with an improvement ratio of $\approx 125 \%$ in $\S 7$.

SLP has been widely studied in the early days of program optimization $[1,3,21]$. Recently, Boyar et al. revisited SLP with the XOR operator $[17,18]$ to optimize (compress) bitmatrices used in the field of cryptography, such as the AES S-box [85, 95, 97]. Their approach is based on Paar's heuristic [78], which is almost the same as RePAIR [67]. Previous works for cryptography [18, 60, 85, 97] focus on reducing the XORs in such special SLPs even if spending several days on one SLP. Indeed, the proposed heuristics run in exponential time for aggressive optimization. However, for RS(10, 4), as we will see in $§ 7$, we need to optimize 1002 SLPs for encoding and decoding. On the basis of this difference, we propose the new heuristics XorRePAIR running in polynomial time. Although the work of Boyar et al. inspired the authors, we emphasize that they did not consider memory and cache optimization because their goal was to compress bitmatrices.

Hong and Kung proposed the red-blue pebble game [47] to model and study the transfer cost between fast and slow devices. This game has been used to analyze a fixed algorithm and program, rather than for optimization. There is a recent remarkable work by Kwasniewski et al. where they used the pebble game to prove the near-optimality of their fixed MM algorithms [61]. Recently, there has been a trend to use the red-blue pebble game for program optimization [23, 35, 80]. Our work is in this direction; indeed, the pebble game is the basis of our cache optimization algorithm.

\section{REDUCING XOR OPERATIONS}

We formally introduce SLP with the XOR operator. To optimize SLP, we employ a compression algorithm, RePAIR, and extend it to XORREPAIR by accommodating a property of XOR. We will measure and compare the performance of REPAIR and XORREPAIR in $§ 7$.

\subsection{Straight-Line Program}

A straight-line program (SLP) is a program without branchings, loops, and procedures $[1,3,21]$. An SLP is a tuple $\langle\mathscr{V}, \mathscr{C}, \vec{s}, \vec{g}, \otimes\rangle$ where $\mathscr{V}$ is a set of variables, $\mathscr{C}$ is a set of constants, $\vec{s}$ is a sequence of instructions (i.e., the body of the program), $\vec{g}$ is a sequence of variables returned by the program, and $\otimes$ is a binary operator. The set of instructions 〈instr〉 is defined by the following BNF:

$$
\begin{aligned}
& \langle\text { instr }\rangle:=\mathscr{V} \leftarrow\langle\text { expr }\rangle \\
& \langle\text { expr }\rangle:=\mathscr{V}|\mathscr{C}|\langle\text { expr }\rangle \otimes\langle\text { expr }\rangle
\end{aligned}
$$

We consider a class of SLPs, XOR SLP [17, 18], whose binary operator only satisfies the associativity $((x \oplus y) \oplus z=x \oplus(y \oplus z))$, commutativity $(x \oplus y=y \oplus x)$, and cancellativity $(x \oplus x \oplus y=y)$ laws. We write $\mathbb{S L P} \oplus$ for the class.

$\mathbb{S L P}_{\oplus}$ is a DSL for XORing byte arrays. For example, the following left $\mathbb{S L P} \oplus$ abstracts the right array program:

$$
\begin{aligned}
& v_{1} \leftarrow a \oplus b ; \quad \mathrm{P}(\mathrm{a}, \mathrm{b}, \mathrm{c}, \mathrm{d}: \text { [byte]) } \\
& \begin{array}{ll}
v_{1} \leftarrow a \oplus b ; & \text { var } \mathrm{v}_{1}=\mathrm{a} \text { xor } \mathrm{b} ; \\
v_{2} \leftarrow b \oplus c \oplus d ; & \operatorname{var} \mathrm{v}_{2}=\text { (b xor } \mathrm{c} \text { ) xor } \mathrm{d} \text {; }
\end{array} \\
& v_{3} \leftarrow v_{1} \oplus v_{2} ; \quad \Leftrightarrow \quad \text { var } v_{3}=v_{1} \text { xor } v_{2} \text {; } \\
& \operatorname{ret}\left(v_{2}, v_{3}, v_{1}\right) \quad \text { return }\left(\mathrm{v}_{2}, \mathrm{v}_{3}, \mathrm{v}_{1}\right) \text {; } \\
& \text { \} }
\end{aligned}
$$

where for the left SLP $\mathscr{V}=\left\{v_{1}, v_{2}, v_{3}\right\}, \mathscr{C}=\{a, b, c, d\}$, and $\vec{g}=$ $\left\langle v_{2}, v_{3}, v_{1}\right\rangle$, and for the array program the infix function xor performs XOR element-wise for input byte arrays. On the basis of this idea, we have the following correspondence:

$$
\begin{aligned}
& \text { constants of } \mathbb{S L P}_{\oplus} \Leftrightarrow \text { program input arrays, } \\
& \text { variables of } \mathbb{S L P}_{\oplus} \Leftrightarrow \text { arrays allocated at runtime. }
\end{aligned}
$$

Calculus on $\mathbb{S L P} \mathbb{P}_{\oplus}$. We consider a set-based semantics where the value of a variable is a set of the constants. We interpret $\oplus$ as the symmetric difference of sets; e.g., $\{a, b\} \oplus\{c, d\}=\{a, b, c, d\}$ and $\{a, b\} \oplus\{a, c\}=\{b, c\}$. This semantics enables us to compute the above example $\mathbb{S L} \mathbb{P} \oplus$ as follows:

\begin{tabular}{l|ccc}
\multicolumn{1}{c|}{ SLP $P$} & $v_{1}$-value & $v_{2}$-value & $v_{3}$-value \\
\hline$v_{1} \leftarrow a \oplus b ;$ & $\{a, b\}$ & & \\
$v_{2} \leftarrow b \oplus c \oplus d ;$ & $\{a, b\}$ & $\{b, c, d\}$ & \\
$v_{3} \leftarrow v_{1} \oplus v_{2} ;$ & $\{a, b\}$ & $\{b, c, d\}$ & $\{a, c, d\}$ \\
$\operatorname{ret}\left(v_{2}, v_{3}, v_{1}\right)$ & & &
\end{tabular}

Notation. We use $\llbracket \cdot \rrbracket$ to denote the returned values of a program; e.g., $\llbracket P \rrbracket=\langle\{b, c, d\},\{a, c, d\},\{a, b\}\rangle$. We use $\#_{\oplus} \cdot$ to denote the size of a program, i.e., the number of XORs; e.g., $\#_{\oplus} P=4$. We use $\mathrm{N} \operatorname{Var}(\cdot)$ to denote the number of variables; e.g., $\mathrm{N} \operatorname{Var}(P)=$ $\left|\left\{v_{1}, v_{2}, v_{3}\right\}\right|=3$ where $|S|$ is the cardinality of a finite set $S$.

\subsection{Shortest SLP Problem}

We formalize our first optimization problem.

The shortest $S L \mathbb{P} \oplus$ problem

For a given $P \in \mathbb{S L} \mathbb{P}_{\oplus}$, we find $Q \in \mathbb{S L} \mathbb{P}_{\oplus}$ that satisfies $\llbracket P \rrbracket=$ $\llbracket Q \rrbracket$ and minimizes $\#_{\oplus} Q$.

We cannot solve this problem in polynomial time unless $\mathbf{P}=\mathbf{N P}$ since the NP-completeness of its decision problem version was shown by Boyar et al [18]. They reduced the NP-complete problem Vertex Cover Problem [39] to the above problem.

Example: Minimizing via Cancellation. Let us consider the following three equivalent SLPs:

\begin{tabular}{lll}
$\mathbb{S L P}_{\oplus} P_{0}$ & $\mathbb{S L P}_{\oplus} P_{1}$ & $\mathbb{S L \mathbb { P }} P_{2}$ \\
\hline$v_{1} \leftarrow a \oplus b ;$ & $v_{1} \leftarrow a \oplus b ;$ & $v_{1} \leftarrow a \oplus b ;$ \\
$v_{2} \leftarrow a \oplus b \oplus c ;$ & $v_{2} \leftarrow v_{1} \oplus c ;$ & $v_{2} \leftarrow v_{1} \oplus c ;$ \\
$v_{3} \leftarrow a \oplus b \oplus c \oplus d ;$ & $v_{3} \leftarrow v_{2} \oplus d ;$ & $v_{3} \leftarrow v_{2} \oplus d ;$ \\
$v_{4} \leftarrow b \oplus c \oplus d ;$ & $v_{4} \leftarrow b \oplus c \oplus d ;$ & $v_{4} \leftarrow v_{3} \oplus a ;$ \\
$\operatorname{ret}\left(v_{1}, v_{2}, v_{3}, v_{4}\right)$ & $\operatorname{ret}\left(v_{1}, v_{2}, v_{3}, v_{4}\right)$ & $\operatorname{ret}\left(v_{1}, v_{2}, v_{3}, v_{4}\right)$
\end{tabular}

where $\llbracket P_{0} \rrbracket=\llbracket P_{1} \rrbracket=\llbracket P_{2} \rrbracket, \#_{\oplus} P_{0}=8, \#_{\oplus} P_{1}=5$, and $\#_{\oplus} P_{2}=4$. We notice that, in $P_{2}$, the $\oplus$-cancellativity is effectively used to compute $v_{4}$. Indeed, we can show that there is no $Q$ with $\#_{\oplus} Q<4$ 
and $\llbracket P_{0} \rrbracket=\llbracket Q \rrbracket$ enumerating $Q \in \mathbb{S L} \mathbb{P}_{\oplus}$, Moreover, there is no $Q$ with $\#_{\oplus} Q<5$ and $\llbracket P_{0} \rrbracket=\llbracket Q \rrbracket$ unless using the $\oplus$-cancellativity.

This examples emphasizes the $\oplus$-cancellativity is essential to shorten $\mathbb{S L P}_{\oplus}$. We can also say that $P_{2}$ is $2 \mathrm{x}$ faster than $P_{0}$.

\subsection{Compressing SLP by RePAIR}

Instead of searching the shortest SLPs by tackling the intractable optimization problem, we employ the grammar compression algorithm REPAIR from grammar compression theory as a heuristic.

In the original paper of REPAIR [67], Larsson and Moffat applied a procedure called pairing recursively to compress data (REPAIR stands for recursive pairing). For an SLP $P$ and a pair $(x, y)$ of terms (constants and variables), we replace all the occurrences of the pair in $P$ introducing a fresh variable. Hereafter, we call this step $\operatorname{PAIR}(x, y)$. Let us apply $\operatorname{PAIR}(a, b)$ to the previous $\mathbb{S L I}_{\oplus} P_{0}$.

$$
\begin{array}{lll}
v_{1} \leftarrow a \oplus b ; & & t_{1} \leftarrow a \oplus b ; \\
v_{2} \leftarrow a \oplus b \oplus c ; & v_{2} \leftarrow t_{1} \oplus c ; \\
v_{3} \leftarrow a \oplus b \oplus c \oplus d ; & \operatorname{PAIR}(a, b) & v_{3} \leftarrow t_{1} \oplus c \oplus d ; \\
v_{4} \leftarrow b \oplus c \oplus d ; & & v_{4} \leftarrow b \oplus c \oplus d ; \\
\operatorname{ret}\left(v_{1}, v_{2}, v_{3}, v_{4}\right) & & \operatorname{ret}\left(t_{1}, v_{2}, v_{3}, v_{4}\right)
\end{array}
$$

It replaces all $a \oplus b$ with the new variable $t_{1}$ and reduces XORs.

To distinguish variables introduced by PAIR and the others, we use horizontal lines as above. Variables introduced by PAIR, $t_{1}, t_{2}, \ldots$, are called temporals and the others originals; e.g., $t_{1}$ is temporal and $v_{2}, v_{3}, v_{4}$ are original.

To define our version of REPAIR, we need a total order $<$ on terms and extend it to the lexicographic ordering $\sqsubset$ on pairs. In this paper, as an example, we use the total order defined as follows: we order all constants in the alphabetical order and order temporal variables using their generation order: $t_{1} \prec t_{2} \prec \ldots<t_{m}$ where $t_{i}$ is generated before $t_{i+1}$ by PAIR. Furthermore, we require $t<c$ for a temporal variable $t$ and a constant $c$.

Now, we define REPAIR using PAIR as its subroutine.

\section{RePaIR}

loop

(1) If there is no original variable, we terminate.

(2) Otherwise, we choose a pair of terms that most frequently appears in the definitions of original variables (i.e., below the horizontal line). We then apply PAIR with the pair. If there are multiple candidates, we select the smallest one for $\sqsubset$.

Example. Let us apply RePAIR to the above $P_{0}$ omitting ret:

$$
\begin{aligned}
& v_{1} \leftarrow a \oplus b \\
& v_{2} \leftarrow a \oplus b \oplus c ; \quad t_{1} \leftarrow a \oplus b ; \quad t_{1} \leftarrow a \oplus b \\
& v_{3} \leftarrow a \oplus b \oplus c \oplus d ; \stackrel{(a, b)}{\Rightarrow} \quad v_{2} \leftarrow t_{1} \oplus c ; \quad \stackrel{\left(t_{1}, c\right)}{\Rightarrow} \frac{t_{2} \leftarrow t_{1} \oplus c ;}{v_{3} \leftarrow t_{2} \oplus d ;} \\
& v_{4} \leftarrow b \oplus c \oplus d ; \quad v_{4} \leftarrow b \oplus c \oplus d ; \quad v_{4} \leftarrow b \oplus c \oplus d ;
\end{aligned}
$$

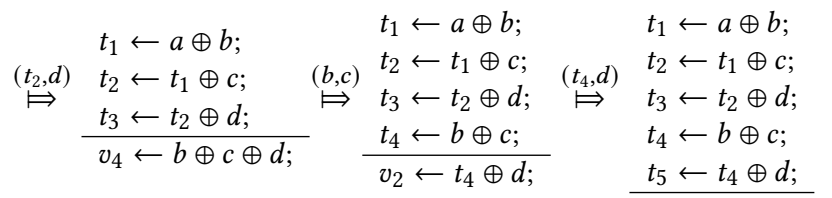

At the first step, the pairs $(a, b)$ and $(b, c)$ appear three times, and we choose $(a, b)$ because $(a, b) \sqsubset(b, c)$. The rest of the parts are processed in the same way. We note that REPAIR reduces eight XORs to five, and the obtained SLP equals the previous $P_{1}$.

\subsection{New Heuristic: XorRePaIr}

We extend REPAIR by accommodating the XOR-cancellativity, which is not considered at all in RePAIR.

First, we introduce an auxiliary procedure, $\operatorname{ReBuILD}(v)$, which rewrites the definition of a given original variable $v$ using the values of temporal variables. We also use the auxiliary notation $(w)$ to denote the value of a variable $w$.

REBUILD(v: original variable)

Initialize: Let $r e m:=(v)$ and $\mathcal{S}:=\emptyset$. rem denotes a set of constants to be eliminated by XORing existing temporal variables. loop

(1) If we cannot shorten rem (i.e., there is no temporal variable $t$ such that $\mid$ rem $\oplus(t)|<|$ rem $\mid)$, we return rem $\cup \mathcal{S}$ as the new definition of $v$.

(2) Otherwise, we choose a temporal variable $t$ that minimizes $\mid$ rem $\oplus(t) \mid$ and update rem $:=$ rem $\oplus(t)$ and $\mathcal{S}:=\mathcal{S} \cup\{t\}$. If there are multiple candidates $t$, we choose the smallest one for $\prec$.

For example, applying $\operatorname{ReBuILD}\left(v_{4}\right)$ to the following left SLP, we obtain a new equivalent definition $v_{4} \leftarrow a \oplus t_{3}$ :

$$
\begin{aligned}
& \text { (1) Set rem }=\{b, c, d\}=\left(v_{4}\right) \text {. } \\
& t_{1} \leftarrow a \oplus b ; \quad \text { (2) Choose } t_{3} \text { because } \\
& \left.t_{2} \leftarrow t_{1} \oplus c ; \quad t_{1}:|\operatorname{rem} \oplus| t_{1}\right)|=|\{a, c, d\} \mid=3 ; \\
& \left.t_{3} \leftarrow t_{2} \oplus d ; \quad t_{2}: \mid \text { rem } \oplus 0 t_{2}\right)|=|\{a, d\} \mid=2 \text {; } \\
& \left.v_{4} \leftarrow b \oplus c \oplus d ; \quad t_{3}:|r e m \oplus| t_{3}\right)|=|\{a\} \mid=1 \text {; } \\
& \Downarrow \\
& \left\{a, t_{3}\right\} \\
& \text { (3) Set rem }=\{a\} \text { and } \mathcal{S}=\left\{t_{3}\right\} \text {. } \\
& \text { (4) Return }\{a\} \cup\left\{t_{3}\right\} \text { because } \\
& \left|r e m \oplus\left(t_{i}\right)\right|>|\{a\}|(=1) \quad \forall i \in\{1,2,3\} .
\end{aligned}
$$

Augmenting RePaIr with Rebuilt, we obtain XorRePair:

$$
\begin{aligned}
& \text { XORREPAIR = REPAIR + REBUILD } \\
& \text { loop } \\
& \text { (1) and (2) are the same as RePAIR. } \\
& \text { (3) For each original variable } v \text {, if } \operatorname{ReBUILD}(v) \text { is strictly } \\
& \text { smaller than the current definition of } v \text {, we update } v \text {. }
\end{aligned}
$$

Let us apply XorRePair to the example $P_{0}$.

$$
\begin{aligned}
& t_{1} \leftarrow a \oplus b ; \quad t_{1} \leftarrow a \oplus b ; \quad t_{1} \leftarrow a \oplus b ; \\
& \ldots \models \quad t_{2} \leftarrow t_{1} \oplus c ; \quad \stackrel{\operatorname{ReBuILD}}{\models} t_{2} \leftarrow t_{1} \oplus c ; \quad \stackrel{\left(a, t_{3}\right)}{\Leftrightarrow} t_{2} \leftarrow t_{1} \oplus c ; \\
& \frac{t_{3} \leftarrow t_{2} \oplus d ;}{v_{4} \leftarrow b \oplus c \oplus d ;} \quad \Rightarrow \quad \frac{t_{3} \leftarrow t_{2} \oplus d ;}{v_{4} \leftarrow a \oplus t_{3}} \quad \begin{array}{l}
t_{3} \leftarrow t_{2} \oplus d ; \\
t_{4} \leftarrow a \oplus t_{3} ;
\end{array}
\end{aligned}
$$

First, we reach the above left form. Next, we update $v_{4}$ as $v_{4} \leftarrow a \oplus t_{3}$ since $\operatorname{Rebuilt}\left(v_{4}\right)=\left\{a, t_{3}\right\}$ shortens the definition of $v_{4}$. Finally, we perform $\operatorname{PAIR}\left(a, t_{3}\right)$ and obtain the shortest $\mathbb{S L P}_{\oplus}$ with 4 XORs as we have seen in $\S 4.2$. Clearly, XorRePAIR runs in polynomial time.

Related approaches. We note that pairing is sometimes called factoring in the context of common subexpression elimination (CSE) of compiler construction [19]. Combining algebraic properties for simplifying expressions with CSE, like XORRePAIR, has been naturally considered in compiler construction $[2,75]$; however, primal methods to choose terms to be factored are elaborated. We adopt REPAIR to simply implement our compressor. The effectiveness of 
REPAIR is already known in grammar compression [27] and in the context of compressing matrices over $\mathbb{F}_{2}$ for cryptography [60].

\section{REDUCING MEMORY ACCESS}

We reduce memory accesses of SLPs by employing deforestation, an optimization method of functional program [32, 41, 100].

Deforestation has deep theoretical backgrounds [22, 32, 41, 96, 99, 100]; however, we need a trivial idea for SLPs. Let us consider the following SLP and the corresponding program:

$$
\begin{array}{ll}
v_{1} \leftarrow a \oplus b ; & \operatorname{program}(\mathrm{a}, \mathrm{b}, \mathrm{c}, \mathrm{d})\{ \\
v_{2} \leftarrow v_{1} \oplus c ; & \quad \operatorname{var} \text { out }=((\mathrm{a} \text { xor b) xor } \mathrm{c}) \text { xor } \mathrm{d} ; \\
v_{3} \leftarrow v_{2} \oplus d ; & \quad \operatorname{return}(\text { out }) ; \\
\operatorname{ret}\left(v_{3}\right) & \}
\end{array}
$$

As the reader might notice, program makes two intermediate byte arrays, which correspond to $v_{1}$ and $v_{2}$. Since these intermediate arrays are immediately released, we would like to eliminate them. To this end, we rewrite program to the following one fusing XORs:

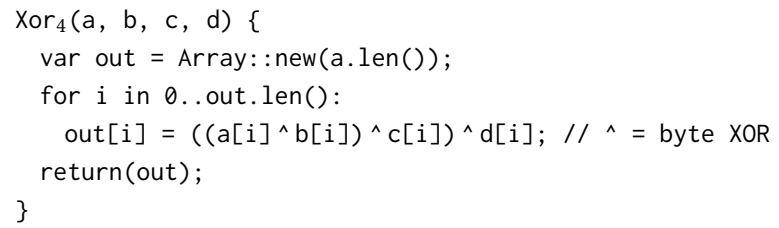

Xor ${ }_{4}$ does not only generate intermediate arrays, but also reduces memory accesses. For $N$-bytes arrays $A$ and $B, A$ xor $B$ invokes $3 N$ memory accesses (loading $A$ and $B$, and writing the XORed result); thus, program invokes $9 N$ memory accesses for $N$-bytes arrays. On the other hand, Xor $_{4}$ invokes $5 \mathrm{~N}$ memory accesses.

Now, we augment SLPs with variadic XOR operators to formalize fused XORs, like Xor ${ }_{4}$. We note that no SLP faithfully reflects Xor ${ }_{4}$ since the formalization of SLPs only admits binary operators.

\subsection{MultiSLP and Memory Accessing Problem}

We extend $S L \mathbb{P}_{\oplus}$ to $\mathbb{S L P}_{\vec{\oplus}}$ by accommodating variadic XORs $\bigoplus(\vec{t})$. $\mathbb{S L P}_{\vec{\oplus}}$ can represent the above Xor $_{4}$ as follows:

$$
\text { 1) } v \leftarrow \bigoplus(a, b, c, d) ; \quad \text { 2) } \operatorname{ret}(v) \text {; }
$$

We also impose on $\operatorname{SLP}_{\vec{\oplus}}$ that there is no nested XORs; indeed, we can remove them as $v \leftarrow\left(x_{1} \oplus x_{2}\right) \oplus x_{3} \Leftrightarrow v \leftarrow \bigoplus\left(x_{1}, x_{2}, x_{3}\right)$.

To formalize our memory access optimization problem, we define the number of memory accesses $\#_{M}(P)$ for $P \in \mathbb{S L I}_{\vec{\oplus}}$ as follows:

$$
\#_{M}(P)=\sum\left\{n+1: v \leftarrow \bigoplus\left(t_{1}, t_{2}, \ldots, t_{n}\right) \in P\right\} .
$$

\footnotetext{
The minimum memory access problem

For an $P \in \mathbb{S L P}_{\oplus}$, we find $Q \in \mathbb{S L}_{\vec{\oplus}}$ that satisfies $\llbracket P \rrbracket=\llbracket Q \rrbracket$ and minimizes ${ }_{M}(Q)$.
}

THEOREM 1. The minimum memory access problem cannot be solved in polynomial time unless $\boldsymbol{P}=\mathbf{N P}$.

This problem is also intractable as the same as the shortest $\mathbb{S L P} \oplus$ We prove the intractability in Appendix §A by reducing the Vertex Cover Problem (VCP) to the above one. Although using the VCP is the same as the construction given by Boyar et al. [18] to prove the intractability of the shortest $\mathbb{S L P} \oplus$ problem, we need to deeply analyze $\mathbb{S L P}_{\oplus}$ and $\mathbb{S L P}_{\vec{\oplus}}$ because a normalization from SLPs to SLPs of the binary form as follows, which is the key in the proof of [18], does not work well for $\operatorname{SLP}_{\vec{\oplus}}$ and $\#_{M}$ :

$$
v \leftarrow a \oplus b \oplus c ; \quad \Rightarrow \begin{aligned}
& v^{\prime} \leftarrow a \oplus b ; \\
& v \leftarrow v^{\prime} \oplus c ;
\end{aligned}
$$

The above normalization to the binary form does not change $\#_{\oplus}$; however, it increases ${ }_{M}(4 \rightarrow 6)$. This normalization brought a significantly useful syntactic property on $\mathbb{S L P}_{\oplus}$ in [18]. Since we cannot count on such the property, in Appendix §A, we give a more detailed and elaborated construction.

\subsection{XOR Fusion}

We propose a heuristic, XOR fusion, which reduces memory accesses of a given $\mathbb{S L P}_{\oplus}$ by transforming it to $\mathbb{S L P}_{\vec{\oplus}}$.

\section{XOR fusion}

Repeatedly applying the procedure that if there is a variable $v$ used just once in the program, we unfold $v$ as follows:

$$
\begin{aligned}
& v \leftarrow \bigoplus\left(t_{1}, t_{2}, \ldots, t_{n}\right) ; \quad \Leftrightarrow \quad v^{\prime} \leftarrow \bigoplus\left(\ldots, t_{1}, t_{2}, \ldots, t_{n}, \ldots\right) ; \\
& v^{\prime} \leftarrow \bigoplus(\ldots, v, \ldots) ;
\end{aligned}
$$

The following is an example of the XOR fusion:

$$
\begin{aligned}
& v_{1} \leftarrow a \oplus b ; \\
& v_{2} \leftarrow v_{1} \oplus c ; \quad \Leftrightarrow \begin{array}{l}
v_{2} \leftarrow \bigoplus(a, b, c) ; \\
v_{3} \leftarrow v_{2} \oplus d ;
\end{array} \quad \Leftrightarrow v_{3} \leftarrow v_{2} \oplus d ;
\end{aligned} \quad \Rightarrow \bigoplus(a, b, c, d) ;
$$

The fusion reduces memory accesses, and the following holds.

Theorem 2. Let $P$ be an $\mathbb{S L P}_{\vec{\oplus}}$, and $Q$ be an $\mathbb{S L P}_{\vec{\oplus}}$ obtained by applying the XOR fusion to $P$. Then, $\#_{M}(Q)<\#_{M}(P)$ holds.

Why do not unfold variables used more than once? Let us consider the following three SLPs where $A$ is a source SLP, $B$ is obtained one by compressing $A$, and $C$ is obtained by fusing $A$.

$$
\begin{aligned}
& A: \quad v_{2} \leftarrow a \oplus b \oplus c \oplus d \oplus e \oplus f \\
& v_{3} \leftarrow a \oplus b \oplus c \oplus d \oplus e \oplus g ; \quad \text { compress } v_{1} \leftarrow \bigoplus(a, b, c, d, e) \text {; } \\
& \downarrow \text { fuse } \backslash B: v_{2} \leftarrow v_{1} \oplus f \text {; } \\
& C: \quad v_{2} \leftarrow \bigoplus(a, b, c, d, e, f) ; \quad \leftarrow^{-}-{ }^{-} \text {disallow } \quad v_{3} \leftarrow v_{1} \oplus g \text {; } \\
& v_{3} \leftarrow \bigoplus(a, b, c, d, e, g)
\end{aligned}
$$

where $\#_{M}(A)=30$ since one XOR issues three accesses, $\#_{M}(B)=12$, and $\#_{M}(C)=14$. Therefore, if we would allow to unfold $v_{1}$ in $B$, then fusing increases memory accesses. In other words, the fusion without the restriction uncompresses a given SLP too much.

On the other hand, this situation suggests that uncompressed but fused SLP may run quickly in the real situation. Since compressing introduces extra variables as above, it may bring terrible effects on cache. In the next section §6, we will consider cache optimization. Furthermore, we will compare the coding throughputs of directly fused SLPs and fully optimized (compressed, fused, and cache optimized) ones in §7.

\section{REDUCING CACHE MISSES}

We proposed the XOR fusion to reduce memory accesses in the previous section. We now go one step further and reduce cache 
misses. To this end, we first review a classical cache optimization technique, blocking, and then formalize our cache optimization problem on the basis of the (red-blue) pebble game [47, 91]. We see that our optimization problem cannot be solved in polynomial-time (unless $\mathbf{P}=\mathbf{N P}$ ) and provide polynomial-time heuristics.

\subsection{Blocking Technique for Cache Reusing}

Since the size of a CPU cache is small with compared to that of main memory, we can only put a few arrays if a given data to be encoded is large. For example, if the size of $\mathrm{L} 1$ cache is $32 \mathrm{~KB}$, which is a typical L1 cache size, and a user encodes $1 \mathrm{MB}$ data on $\mathbf{R S}(10,4)$, the input data is divided into $8 \cdot 10$ arrays of $\frac{1 \mathrm{MB}}{80} \approx 12 \mathrm{~KB}$; therefore, the cache can only hold two arrays at once, and thus the cache performance becomes poor.

To hold many arrays in cache at once, we use the established cache optimization technique blocking, which splits large arrays into small blocks introducing a loop. Let us perform blocking for the following example, where we split arrays into arrays of $\mathcal{B}$ bytes.

\begin{tabular}{|c|c|}
\hline Original Program & Blocked One (Blocksize is $\mathcal{B}$ ) \\
\hline $\operatorname{ain}(\ldots)\{$ & $\begin{array}{l}\operatorname{main}(\ldots)\{ \\
\quad \text { for } i \text { in } 0 \ldots(A \cdot \operatorname{len}() / \mathcal{B})\end{array}$ \\
\hline $\mathrm{v}_{1}=\operatorname{xor}(\mathrm{A}, \mathrm{B})$; & $\mathrm{v}_{1}^{[i]}=\operatorname{xor}\left(\mathrm{A}^{[i]}, \mathrm{B}^{[i]}\right)$ \\
\hline$v_{2}=\operatorname{xor}(C, D)$ & $v_{2}^{[i]}=\operatorname{xor}\left(C^{[i]}, D^{[i]}\right) ;$ \\
\hline$v_{3}=\operatorname{xor}\left(V_{1}, E, F\right)$ & $v_{3}^{[i]}=\operatorname{xor}\left(v_{1}^{[i]}, E^{[i]}, F^{[i]}\right) ;$ \\
\hline$v_{4}=\operatorname{xor}\left(v_{3}, G, A\right)$ & $\mathrm{v}_{4}^{[i]}=\operatorname{xor}\left(\mathrm{v}_{3}^{[i]}, \mathrm{G}^{[i]}, \mathrm{A}^{[i]}\right) ;$ \\
\hline$v_{5}=\operatorname{xor}\left(v_{1}, v_{3}, v_{4}\right)$; & $v_{5}^{[i]}=\operatorname{xor}\left(v_{1}^{[i]}, v_{3}^{[i]}, v_{4}^{[i]}\right)$ \\
\hline$\left., v_{4}, v_{5}\right)$ & return $\left(v_{2}, v_{4}, v_{5}\right)$ \\
\hline
\end{tabular}

where $X^{[i]}$ is the $i$-th $\mathcal{B}$-bytes block of an array $X$; i.e., $X^{[0]}=$ $X[0 . . \mathcal{B}], X^{[1]}=X[\mathcal{B} . .2 \mathcal{B}]$, and so on.

Measures of Cache Efficiency. We consider two measures of cache efficiency. As the first measure, we consider the minimum cache capacity CCap to avoid cache reloading while computing a given program. It is called cache reloading to load a certain block that is once spilled from cache from memory to cache again. If we can transform a given program $P$ to an equivalent one $Q$ with $\operatorname{CCap}(Q)<\operatorname{CCap}(P)$, then we can say $Q$ is more cache efficient. As the second measure, we consider IOcost the total number of I/O transfers between cache and memory. To formalize these measures, we augment SLPs with abstract cache memory.

\subsection{SLP Augmented with Abstract LRU Cache}

To formalize measures for cache efficiency, hereafter we use SLPs to represent the inside of the loop introduced by the blocking technique by forgetting indices. For example, the above program is rewritten as the following SLP $P_{e g}$ :

$$
\begin{array}{lll} 
& \text { 1) } v_{1} \leftarrow A \oplus B ; & \text { 2) } v_{2} \leftarrow C \oplus D ; \\
P_{e g}: & \text { 3) } v_{3} \leftarrow \bigoplus\left(v_{1}, E, F\right) ; & \text { 4) } v_{4} \leftarrow \bigoplus\left(v_{3}, G, A\right) ; \\
\text { 5) } v_{5} \leftarrow \bigoplus\left(v_{1}, v_{3}, v_{4}\right) ; & \text { 6) } \operatorname{ret}\left(v_{2}, v_{4}, v_{5}\right) ;
\end{array}
$$

We introduce notations to operate cache through SLPs.

Computation with Cache. We simply consider a cache $C$ as an ordered sequence of blocks, $C=\beta_{1}, \beta_{2}, \ldots, \beta_{n}$, where each block $\beta_{i}$ is just a variable or constant. The rightmost (resp. leftmost) block represents the most (resp. least) recently used element.

Let us consider to execute an XOR $v \leftarrow \bigoplus\left(t_{1}, t_{2}, \ldots, t_{k}\right)$. We require $\left\{t_{1}, \ldots, t_{k}, v\right\} \subseteq C$ and then change $C$ in the following steps:

(1) For the arguments, in the order $i=1,2, \ldots, k$, we load $t_{i}$ to $C$ if $t_{i} \notin C$ or update the position of $t_{i}$ if $t_{i} \in C$;

(2) We then allocate $v$ in $C$ if $v \notin C$ or update the position of $v$ if $v \in C$.

If the cache is full and there is no room for loading or allocating, we evict the LRU (least recently used) element in the cache. This eviction corresponds to spilling or writing-back a cached block to memory. It corresponds to the typical cache replacement policy LRU replacement policy [46].

Example. Let us run our example SLP $P_{e g}$ with a 10-capacity cache. For the first XOR, we load $A$ and then $B$ and finally allocate $v_{1}$. These operations change $C$ as follows:

$$
\text { empty } \stackrel{A}{\Rightarrow} A \stackrel{B}{\Rightarrow} A B \stackrel{v_{1}}{\rightarrow} A B v_{1}
$$

where we use $\stackrel{\bullet}{\Rightarrow}$ to denote a loading from memory and $\stackrel{\bullet}{\circ}$ to an allocation in the cache.

For the second XOR, we load $C$ and $D$ and allocate $v_{2}$ as follows:

$$
A B v_{1} \stackrel{C}{\Rightarrow} \stackrel{D}{\Rightarrow} \stackrel{v_{2}}{\rightarrow} A B v_{1} C D v_{2} .
$$

For the third XOR, we update the position of $v_{1}$ in the cache, load $E$ and $F$, and allocate $v_{3}$ :

$$
A B v_{1} C D v_{2} \stackrel{v_{1}}{\longrightarrow} \stackrel{E}{\Rightarrow} \stackrel{F}{\Rightarrow} \underset{v_{3}}{\rightarrow} A B C D v_{2} v_{1} E F v_{3}
$$

where we use $\dot{\rightarrow}$ to denote a position update in the cache.

For the fourth XOR, fetching the arguments $\left(v_{3}, G\right.$, and $A$ ) changes $C$ as follows:

$$
A B C D v_{2} v_{1} E F v_{3} \stackrel{v_{3}}{\rightarrow} \stackrel{G}{\Rightarrow} \stackrel{A}{\rightarrow} B C D v_{2} v_{1} E F v_{3} G A .
$$

Since the fetch makes the cache $C$ full, we evict the LRU element $B$ and then allocate $v_{4}$ as follows:

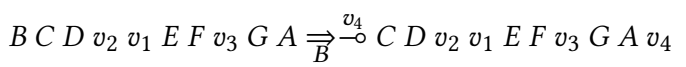

where we write $\Rightarrow$ for evictions from the cache to memory.

Finally, we change $C$ as follows in the fifth XOR:

$C D v_{2} v_{1} E F v_{3} G A v_{4} \stackrel{v_{1}}{\longrightarrow} \stackrel{v_{3}}{\longrightarrow} \stackrel{v_{4}}{\longrightarrow} \underset{C}{\longrightarrow} \stackrel{v_{5}}{\rightarrow} D v_{2} E F G A v_{1} v_{3} v_{4} v_{5}$.

Now, we introduce two notations for the cache efficiency of SLPs.

$\operatorname{CCap}(P: \operatorname{SLP})$

$\mathrm{CCap}(P)$ denotes the minimum cache capacity where we can run $P$ without cache reloading.

We can confirm $\operatorname{CCap}\left(P_{e g}\right)=10$. Indeed, if we use the cache of capacity 9, we need to replace $A$ for $G$ in the fourth XOR $v_{4} \leftarrow$ $\bigoplus\left(v_{3}, G, A\right)$, and this replacement leads to reloading $A$ as follows:

$$
A B C D v_{2} v_{1} E F v_{3} \underset{A}{\stackrel{G}{\vec{B}}} \stackrel{A}{\Rightarrow} C D v_{2} v_{1} E F v_{3} G A
$$

where we write $\underset{x}{\vec{x}}$ for the replacement that evicts $x$ and loads $y$.

We also consider the number of $\mathrm{I} / \mathrm{O}$ transfers required by SLPs. 
IOcost $(P:$ SLP, $c$ : cache capacity)

IOcost $(P, c)$ denotes the number of I/O transfers issued while running $P$ with a cache of $c$-capacity. There are two kinds of I/O transfers; transfers from cache to memory, $\stackrel{\bullet}{\Rightarrow}$, and transfers from memory to cache, $\Rightarrow$.

It is clear that IOcost $\left(P_{e g}, 10\right)=7($ of $\stackrel{\bullet}{\Rightarrow})+2($ of $\Rightarrow)=9$. This measure is useful when cache capacity is determined by hardware. For example, it is one of the standard parameter on recent CPUs that cache size is $32 \mathrm{~KB}$ and cache block size is $64 \mathrm{~B}$. The cache of such CPUs can hold 512 blocks maximally, and thus we optimize IOcost $(P, 512)$.

Hereafter, as an example, we consider that the cache can holds eight blocks maximally. We can easily check IOcost $\left(P_{\text {eg }}, 8\right)=13$ running $P_{e g}$ with the cache of capacity 8.

\subsection{Optimizing SLP via Register Allocation}

To reduce CCap and IOcost, we try register allocation by identifying cache (resp. memory) of our setting as registers (resp. memory) of the usual register allocation setting. Register allocation basically consists from three phases [9, 20, 25, 26, 40]: (1) the register assignment phase where we rename variables of a given program so that it has smaller variables; (2) the register spilling phase where we insert instructions to move the contents of registers to/from memory if variables are many than actual registers; (3) the register coalescing phase where we merge variables that has the same meaning in the syntactic or semantic way.

Using the standard graph-coloring register assignment algorithm, we can obtain the following SLP from $P_{e g}$ :

$$
\begin{array}{rll}
\text { 1) } v_{1} \leftarrow A \oplus B ; & \text { 2) } v_{2} \leftarrow C \oplus D ; \\
P_{\text {reg }}: & \text { 3) } v_{3} \leftarrow \bigoplus\left(v_{1}, E, F\right) ; & \text { 4) } v_{4} \leftarrow \bigoplus\left(v_{3},\right. \\
\text { 5') } v_{1} \leftarrow \bigoplus\left(v_{1}, v_{3}, v_{4}\right) ; & \text { 6) } \operatorname{ret}\left(v_{2}, v_{4}, v_{1}\right) ;
\end{array}
$$

Unlike 5) of $P_{e g}$, in $\left.5^{\prime}\right)$, we store the result $\bigoplus\left(v_{1}, v_{3}, v_{4}\right)$ to $v_{1}$ instead of $v_{5}$ of $P_{e g}$ since $v_{1}$ is no more needed after $5^{\prime}$ ).

Although register assignment reduces variables, $\mathrm{N} \operatorname{Var}\left(P_{\text {reg }}\right)=4$, and $\mathrm{I} / \mathrm{O}$ transfers, IOcost $\left(P_{\text {reg }}, 8\right)=12$, it does not reduce CCap since $\mathrm{CCap}\left(P_{e q}\right)=\mathrm{CCap}\left(P_{\text {reg }}\right)=10$. We note that register spilling is useless since the LRU replacement disallows to select cached elements to be evicted. Register coalescing also does not make any sense at least in the above example. These tell that the cache optimization for SLPs by register allocation is quite limited.

Below we employ another approach, where we rearrange statements and arguments in SLPs. It should be noted that program rearrangement or (re)scheduling is beyond register allocation.

Although register allocation on SLPs is not so powerful as above, it enjoys the following properties:

- The register assignment problem of SLPs can be solved in polynomial time. This comes from the relatively new result that the register assignment of programs in the SSA (static single assignment) form is tractable [14, 44, 81]. A program is in the SSA form if each variable is assigned exactly once [6, 33, 87]. Since there is no branching in SLPs, we can easily convert SLPs to SSA SLPs; thus, the register assignment problem for SLPs is tractable. Of course, the problem for general programs is intractable [26].
- The register coalescing problem for SSA SLPs is also tractable; indeed, the variable coalescing operation does not increase the required number of registers. The problem for general programs is intractable $[15,43]$.

- Register spilling is useful in the case where we can select elements to be evicted from the abstract cache. Then, on SSA SLPs, if each variable is used at most once, the minimum cost register spilling problem for SSA SLPs can be solved in polynomial time [16]. Without the constraint, the problem becomes intractable [37].

\subsection{Optimizing SLP via Pebble Game}

We employ the classical tool of program analysis pebble game to make a given SLP cache friendly. On this setting, we do not only rename variables as well as register assignment does but also reorder the entire program. We first introduce computation graphs, which are arenas of the pebble game, and then review the pebble game.

Computation Graph. We use directed acyclic graphs (DAGs) to represent the value dependencies of SLPs:

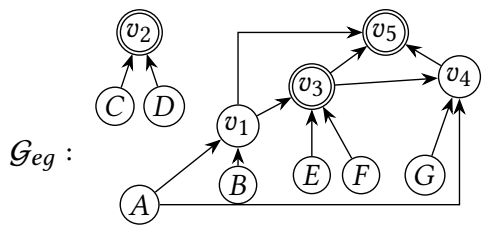

This DAG corresponds to our example $P_{e g}$ in the following sense. Each leaf node (node with no children) represents the constant of the same name. Each inner node (node with children) represents the value obtained by XORing all children; thus, the inner node $v_{1}$ means $A \oplus B, v_{3}$ means $A \oplus B \oplus E \oplus F$, and so on. Computation graphs (CGs) are DAGs with double-circled nodes, goal nodes, which mean values returned by programs. It should be noted that CGs differ from interference graphs, which are used in register allocation [9, $20,25,26,40]$.

Pebble Game. Let $\mathcal{G}$ be a CG. As the initialization step, for each leaf node $\ell$, we put the same name pebble on $\ell$. To represent this, we write $\mathcal{G}(\ell)=\ell$. We win a game if every goal node has a pebble. To achieve this, we pebble inner nodes using the following rules:

- At each turn, the player proposes an instruction of the form

$$
n: p \leftarrow \bigoplus\left(p_{1}, p_{2}, \ldots, p_{k}\right)
$$

where $n$ is a node of $\mathcal{G}, p$ and $p_{i}$ are pebbles, and $\left\{n_{i}\right.$ : $\mathcal{G}\left(n_{i}\right)=p_{i}$ \} equals to $n$ 's children.

- If $p$ is a new pebble, we put $p$ on $n$ so that $\mathcal{G}(n)=p$.

- Otherwise, $p$ is in a node $m$, we move $p$ from $m$ to $n$.

- To avoid computing a single node multiple times, we are disallowed to put or move a pebble to a node once pebbled.

Our pebble game on CGs is equivalent to the standard pebble game of Sethi [90, 91]. Especially, it is equivalent to the red-blue pebble game of Hong and Kung [47] that our game with the measure IOcost and the abstract cache $C$ where we can select elements to be evicted from $C$ instead of the LRU rule. 
Example. Let us consider the following winning strategy (with a return statement) of the above CG $\mathcal{G}_{e g}$ :

$$
\begin{array}{lll}
\text { 1) } v_{1}: p_{1} \leftarrow B \oplus A ; & \text { 2) } v_{3}: p_{2} \leftarrow \bigoplus\left(E, F, p_{1}\right) ; \\
Q: & \text { 3) } v_{4}: p_{3} \leftarrow \bigoplus\left(A, G, p_{2}\right) ; & \text { 4) } v_{5}: p_{1} \leftarrow \bigoplus\left(p_{1}, p_{2}, p_{3}\right) ; \\
\text { 5) } v_{2}: p_{3} \leftarrow C \oplus D ; & \text { 6) } \operatorname{ret}\left(p_{3}, p_{2}, p_{1}\right) ;
\end{array}
$$

This is better than $P_{\text {reg }}$ at all the parameters since $N \operatorname{Var}(Q)=3$, $\operatorname{CCap}(Q)=5$, and $\operatorname{IOcost}(Q, 8)=9$. For example, we can easily confirmed $\operatorname{CCap}(Q)=5$ as follows:

$$
\begin{aligned}
& \text { empty } \stackrel{B}{\Rightarrow} \stackrel{A}{\Rightarrow} \stackrel{p_{1}}{\Rightarrow} B A p_{1} \stackrel{E}{\Rightarrow} \stackrel{F}{\Rightarrow} \stackrel{p_{1}}{\longrightarrow} \frac{p_{2}}{B} A E F p_{1} p_{2} \stackrel{A}{\rightarrow} \stackrel{G}{\vec{E}} \stackrel{p_{2}}{\longrightarrow} \frac{p_{3}}{F} \\
& p_{1} A G p_{2} p_{3} \stackrel{p_{1}}{\longrightarrow} \stackrel{p_{2}}{\longrightarrow} \stackrel{p_{3}}{\longrightarrow} \stackrel{p_{1}}{\longrightarrow} A G p_{2} p_{3} p_{1} \stackrel{C}{\vec{A}} \frac{D}{\vec{G}} \stackrel{p_{3}}{\longrightarrow} p_{2} p_{1} C D p_{3}
\end{aligned}
$$

where $\underset{x}{\mathrm{O}}$ means the replacement that evicts $x$ and allocates $y$.

The pebble game immediately implies the following property.

Proposition 1. Let $\mathcal{W}$ be a winning strategy of the CG of an SLP $P$. Forgetting the node information from $W$ and adding the adequate return statement, we can obtain an $S L P Q_{\mathcal{W}}$ such that $\llbracket P \rrbracket=\llbracket Q_{\mathcal{W}} \rrbracket$.

Notation: Let $P$ and $Q$ be $\operatorname{SLP}_{\vec{\oplus}}$. If $Q$ is obtained from a winning strategy of the CG of $P$ in the above manner, we write $P \vdash Q$.

\subsection{Intractability of Optimization Problems}

Introducing the pebble game is not only useful for cache optimization but also useful to correctly refer established results of compiler construction and program analysis.

Theorem 3. Let $P$ be an $\mathbb{S L P}_{\vec{\oplus}}$. All the following optimization problems cannot be solved in polynomial-time unless $\boldsymbol{P}=\mathbf{N P}$ :

(1) Finding $Q \in \mathbb{S L P} \mathbb{P}_{\vec{\oplus}}$ that satisfies $P \vdash Q$ and minimizes $N \operatorname{Var}(Q)$.

(2) Finding $Q \in \mathbb{S L P} \vec{\oplus}$ that satisfies $P \vdash Q$ and minimizes $\operatorname{CCap}(Q)$.

(3) For a given cache capacity c, finding $Q \in \mathbb{S L I}_{\vec{\oplus}}$ that satisfies $P \vdash Q$ and minimizes $\operatorname{IOcost}(Q, c)$.

In order to show the intractability of Problems (1) and (2), we can use the NP-completeness of the standard pebble game shown by Sethi $[90,91]$. Sethi reduced the classical NP-complete problem 3SAT [30] to the decision problem of the standard pebble game. Problem (1) is the optimizing version of the decision problem of the standard pebble game; hence, it is intractable. Although Problem (2) seems a problem involved in cache, it is a problem of the standard pebble game rather than the pebble game with cache. Indeed, if we could select pebbles to be evicted instead of the LRU rule, Problems (1) and (2) are essentially equivalent. Even if we follow the LRU rule, the construction given by Sethi in [91] also works well; therefore, Problem (2) is intractable.

On the other hand, Problem (3) should be analyzed using the pebble game augmented with cache; namely, we use the red-blue pebble game of Hong and Kung [47]. We can choose nodes to be evicted from the cache on the ordinal formalization of the red-blue pebble game. The intractability of Problem (3) on the red-blue pebble game was already shown in [35, 80]. In [80], Papp and Wattenhofer used the classic NP-complete problem Hamiltonian path problem [39, 58] and succeeded in providing a simple NP-completeness proof. Fortunately, we can directly apply the construction of Papp and Wattenhofer to Problem (3) in our setting with the LRU eviction rule.
It is worth noting that Problem (1) and its variant can be efficiently solved when playing the pebble game on trees rather than DAGs $[36,69,71,76,89,92]$.

\subsection{Two Scheduling Heuristics}

We consider two simple heuristics for solving the pebble game since our interested problems are intractable. More technically, it is known that those problems are hard to approximate [34, 80].

DFS-based algorithm. Our first heuristic visits the nodes of a given $C G$ in the postorder traversal.

Let us see how our heuristic works for our CG $\mathcal{G}_{e g}$. We need to decide which root node is visited first; here, we choose $v_{2}$ on the basis of the total ordering $<$ defined in $\S 4.3$ since $v_{2} \prec v_{5}$. We then visit the children $C$ and $D$ in this order since $C<D$. Using $<$ as the tie-breaker, we make the following postorder traversing:

$C \rightarrow D \rightarrow v_{2} \rightarrow A \rightarrow B \rightarrow v_{1} \rightarrow E \rightarrow F \rightarrow v_{3} \rightarrow G \rightarrow v_{4} \rightarrow v_{5}$.

On the basis of this order, we generate a winning strategy as follows:
1) $v_{2}: p_{1} \leftarrow C \oplus D$
2) $v_{1}: p_{2} \leftarrow A \oplus B$
$Q_{\mathrm{DFS}}:$ 3) $v_{3}: p_{3} \leftarrow \bigoplus\left(p_{2}, E, F\right)$;
4) $v_{4}: p_{4} \leftarrow \bigoplus\left(p_{3}, A, G\right)$;
5) $v_{5}: p_{4} \leftarrow \bigoplus\left(p_{2}, p_{3}, p_{4}\right)$;
6) $\operatorname{ret}\left(p_{1}, p_{3}, p_{4}\right)$;

Our pebble assigning policy is simple. If we have a pebble on $G$ that can move, we reuse it; otherwise, we put a fresh pebble. It can be verified that $\mathrm{NVar}\left(Q_{\mathrm{DFS}}\right)=4, \operatorname{CCap}\left(Q_{\mathrm{DFS}}\right)=7$, and $\operatorname{IOcost}\left(Q_{\mathrm{DFS}}, 8\right)=10$.

Bottom-up greedy algorithm. Our next heuristic is a greedy one. Unlike the above DFS-based algorithm, this heuristic requires a parameter $c$ corresponding to cache capacity.

(i) Choose a computable node $n$, whose children have pebbles, that maximises the ratio $\frac{|H|}{|C|}$ where $C$ are the children of $n$ and $H \subseteq C$ are the children whose pebble in the cache.

(ii) Access $H$ and then access $C$.

(iii) If there is a movable cached pebble, we move it to $n$. Otherwise, we use a movable pebble or allocate a fresh pebble.

Here we again use $<$ as the tie-breaker.

We revisit the CG $\mathcal{G}_{e g}$ as follows. On the initial state, $v_{1}$ and $v_{2}$ are ready with $\frac{|\emptyset|}{|\{A, B\}|}=\frac{0}{2}$ for $v_{1}$ and $\frac{|\emptyset|}{|\{C, D\}|}=\frac{0}{3}$ for $v_{2}$. We choose $v_{1}$ since $v_{1} \prec v_{2}$, and the generated statement changes $C$ as the following right:

$$
v_{1}: p_{1} \leftarrow A \oplus B ; \quad \text { empty } \stackrel{A}{\Rightarrow} \stackrel{B}{\Rightarrow} \stackrel{p_{1}}{\circ} A B p_{1} .
$$

Next, we choose $v_{3}$ since $v_{3}: \frac{\left|\left\{v_{1}\right\}\right|}{\left|\left\{v_{1}, E, F\right\}\right|}=\frac{1}{3}$ and $v_{2}: \frac{|\emptyset|}{|\{C, D\}|}=\frac{0}{2}$, and compute $v_{3}$ with a fresh pebble $p_{2}$. Repeating this procedure, we obtain the following sequences and an SLP $Q_{\text {greedy: }}$ :

$$
\begin{array}{ll}
v_{3}: p_{2} \leftarrow \bigoplus\left(p_{1}, E, F\right) ; & \ldots \stackrel{p_{1}}{\longrightarrow} \stackrel{E}{\Rightarrow} \stackrel{F}{\Rightarrow} \stackrel{p_{2}}{\rightarrow} A B p_{1} E F p_{2}, \\
v_{4}: p_{3} \leftarrow \bigoplus\left(p_{2}, A, G\right) ; & \cdots \stackrel{p_{2}}{\longrightarrow} \stackrel{A}{\longrightarrow} \stackrel{G}{\Rightarrow} \stackrel{p_{3}}{\rightarrow} B p_{1} E F p_{2} A G p_{3}, \\
v_{5}: p_{1} \leftarrow \bigoplus\left(p_{1}, p_{2}, p_{3}\right) ; & \cdots \stackrel{p_{1}}{\longrightarrow} \stackrel{p_{2}}{\longrightarrow} \stackrel{p_{3}}{\longrightarrow} \stackrel{p_{1}}{\longrightarrow} B E F A G p_{2} p_{3} p_{1}, \\
v_{2}: p_{3} \leftarrow C \oplus D ; & \cdots \stackrel{C}{\vec{B}} \stackrel{D}{\vec{E}} \stackrel{p_{3}}{\longrightarrow} \text { EFAG } p_{2} p_{1} C D p_{3} .
\end{array}
$$

It can be verified that $N \operatorname{Var}\left(Q_{\text {greedy }}\right)=3, \operatorname{CCap}\left(Q_{\text {greedy }}\right)=7$, and IOcost $\left(Q_{\text {greedy }}, 8\right)=9$. The scores of NVar and IOcost are optimal. 
This table also says XorRePAir exploits the cancellative property of XOR; but, the difference is minor. It is not surprising; indeed, exponential-time compression heuristics and an algorithm, which corresponds to REPAIR, were already compared in [60] for the application to cryptography, and there was also little difference. These results mean that REPAIR efficiently compresses programs, even though it does not use the cancellativity of XOR. We consider this comes from the robustness of RePair, which also appears in grammar compression when comparing it with other compression algorithms, such as LZ77 and LZ78 [27].

Reducing Memory Access. We see how XORRePAIR and the XOR fusion of $§ 5.1$ reduce memory access $\#_{M}(\cdot)$ :

\begin{tabular}{c|cccc}
$\operatorname{Avg} \%$ & $\frac{\operatorname{Co}(P)}{P}$ & $\frac{\mathrm{Fu}(P)}{P}$ & $\frac{\mathrm{Fu}(\mathrm{Co}(P))}{\mathrm{Co}(P)}$ & $\frac{\mathrm{Fu}(\mathrm{Co}(P))}{P}$ \\
\hline$\#_{M}(\cdot)$ & $40.8 \%$ & $35.1 \%$ & $59.2 \%$ & $24.1 \%$
\end{tabular}

where Co means XorRePAIR, and Fu means the XOR fusion.

The second ratio says that the XOR fusion averagely reduces $\sim 65 \%$ memory accesses for uncompressed SLPs. We can see the other columns in the same way. Therefore, we can tell that XoRREPAIR and the XOR fusion work well independently; furthermore, combining them averagely reduces $\sim 76 \%$ memory accesses on average.

Reducing Variables and Required Cache Size. We consider how the XOR fusion and our DFS-scheduling heuristic averagely affect the two measures of the cache efficiency NVAR and CCap.

\begin{tabular}{|c|c|c|c|c|}
\hline $\operatorname{Avg} \%$ & $\frac{\operatorname{Co}(P)}{P}$ & $\frac{\mathrm{Fu}(P)}{P}$ & $\frac{\mathrm{Fu}(\mathrm{Co}(P))}{\operatorname{Co}(P)}$ & $\frac{\operatorname{DFs}(\mathrm{Fu}(\mathrm{Co}(P)))}{\operatorname{Co}(P)}$ \\
\hline NVAR & $1552 \%$ & $100 \%$ & $38.9 \%$ & $24.5 \%$ \\
\hline$\overline{\mathrm{C}} \overline{\mathrm{Ca}} \overline{\mathrm{p}}$ & $\overline{4} 9 \overline{8} \%$ & $9 \overline{8} . \overline{7} \%$ & $51.2 \%$ & $\overline{4} 0.0 \overline{\%}$ \\
\hline
\end{tabular}

where DFs means our DFS-based scheduling heuristic. We skip using our greedy-scheduling heuristic and the measure IOcost (_,_) since they depend on the cache sizes determined by our block size $64,128, \ldots, 4 K$, and the table including values for of all the cache sizes becomes too large.

The first ratio clarifies that XoRRePAIr significantly degrade cache efficiency. Comparing the third and fourth ratios, we can say the scheduling heuristic certainly improves cache efficiency. Multiplying the first and fourth ratios derives $\frac{\mathrm{CCap}(\operatorname{DFs}(\mathrm{Fu}(\mathrm{Co}(P))))}{\mathrm{CCap}(P)} \sim$ $199 \%$; therefore, we can say that the scheduling heuristic can suppress the side effects of XORREPAIR to some extent.

We consider why XORREPAIR significantly deteriorates NVAR and CCap. It results from the intrinsic behaviors of (XOR)RePAIR; namely, they add many temporal variables without considering cache and register efficiency. The same inefficiency problem was pointed in the early research of program optimization as the weak point of CSE [4].

\subsection{Selecting Adequate Blocksize}

As we have seen in $\S 6$, the block size $\mathcal{B}$ of the blocking technique is an essential optimization parameter. Although small blocks are supposed to enable the cache to hold all blocks, the performance table in $\$ 7.2$ defies our prediction. Here we see additional experiments and think about why the performance on small blocks is not good. Since we have already seen the coding performance of
$P_{\text {enc }}$ on various blocks, we first see the performance of the uncompressed but fused version $P_{\mathrm{enc}}^{+F}$ of $P_{\text {enc }}$ to check whether or not a similar tendency appears.

Case1: Uncompressed but Fused SLP. The following table is the coding throughputs $(\mathrm{GB} / \mathrm{sec})$ of $P_{\text {enc }}^{+F}$ :

\begin{tabular}{c|ccccccc} 
Block size (byte) & 64 & 128 & 256 & 512 & $1 \mathrm{~K}$ & $2 \mathrm{~K}$ & $4 \mathrm{~K}$ \\
\hline intel & 0.87 & 1.73 & 2.85 & 4.08 & 5.29 & 5.78 & 4.36 \\
amd & 1.32 & 2.18 & 3.15 & 3.54 & 3.97 & 4.16 & 3.82
\end{tabular}

where $\operatorname{NVAR}\left(P_{\text {enc }}^{+F}\right)=32$ and $\operatorname{CCap}\left(P_{\text {enc }}^{+F}\right)=88$.

We see there are the same patterns at intel and amd; i.e., $2 K>$ $1 K>4 K>512>256>128>64$. This result again defies our prediction since we need $\mathcal{B} \leq 512$ to avoid cache reloading.

Possible reasons for poor performance of small blocks. The performance problem on small blocks may cause from two sources.

Cache conflicts in cache sets. The first source is cache conflicts in cache sets, and it prevents cache from holding $32 K / \mathcal{B}$ blocks. Generally, the $32 \mathrm{~K}$ bytes cache with 8 cache associativity has $\frac{32 K}{8}=4 K$ cache sets where each cache set can hold 8 cache blocks. Accessing a cache block $b$ whose start address is $\mathcal{A}(b), \mathrm{CPU}$ tries to assign $b$ to the $(\mathcal{A}(b) \bmod 4 K)$-th cache set. If the cache set is full (i.e., it already has 8 cache blocks), CPU evicts the LRU cache block in the set to memory. Therefore, accessing two blocks $b_{1}, b_{2}$ such that $\mathcal{A}\left(b_{1}\right) \equiv_{4 K} \mathcal{A}\left(b_{2}\right)$ may cause an eviction in a cache set.

If we take the $4 K$-alignment strategy (i.e., locate all blocks on addresses divisible by $4 K$ ), the cache holds at most 8 blocks regardless of the size $\mathcal{B}$. To avoid the worst situation, several approaches have been proposed [64, 79]; however, optimally aligning blocks is a hard problem. We use a simple approach as follows: for an SLP whose constants are $c_{0}, c_{1}, \ldots$, we allocate $c_{i}$ so that $\mathcal{A}\left(c_{i}\right) \equiv_{4 K}(i \cdot \mathcal{B})$. We do the same for variables $v_{1}, v_{2}, \ldots$ For example, when $\mathcal{B}=1 K$,

$$
\begin{aligned}
& \mathcal{A}\left(c_{0}\right) \equiv_{4 K} \quad 0, \mathcal{A}\left(c_{1}\right) \equiv_{4 K} 1 K, \mathcal{A}\left(c_{2}\right) \equiv_{4 K} 2 K, \mathcal{A}\left(c_{3}\right) \equiv_{4 K} 3 K, \\
& \mathcal{A}\left(c_{4}\right) \equiv_{4 K} 0, \mathcal{A}\left(c_{5}\right) \equiv_{4 K} 1 K, \ldots
\end{aligned}
$$

This strategy is better than $4 \mathrm{~K}$-alignment since accessing to $c_{i}$ and $c_{j}$ never conflict when $i \not \equiv_{4} j$. In conclusion, the smaller the block size, the more difficult using cache efficiently as expected.

Latency Penalty. The second source of the poor performance may be memory access latency. It is clear that, if $\mathcal{B}$ becomes smaller, then the number of required iteration becomes larger. Therefore, on small blocks, there are many unavoidable block loading caused by changing iterations.

We now focus the memory access latency on modern CPUs. For example, we consider Intel's Haswell microarchitecture [45] released in 2013, and it and its successor are widely used today. Haswell needs about 150 CPU cycles as latency to reach RAM [46, 55]. Even if the CPU pipeline maximally works, we need $150+\frac{\mathcal{B}}{n \cdot 8}$ cycles to load or store a block on an $n$-channel memory. Thus, we need 158-cycles to load a 64-bytes block at once on a single channel memory. If we load a 64-bytes block in eight 8-byte loads, then we need $(151 \times 8)$-cycles. This is the reason why we should load a block from the memory as possible as large. Haswell can load two 32-bytes data from the cache, XOR the two data using AVX2 or AVX512, and store the result 32-bytes to the L1 cache in a single cycle [42]. Thus, we can perform XORing for two blocks of $\mathcal{B}$ bytes 
in the cache in $\frac{\mathcal{B}}{32}$ cycles. When $\mathcal{B}=64$ (resp. $\mathcal{B}=4 K$ ), we can perform xor32 $\sim 75$ (resp. 1) times while loading one block from the memory. In a conclusion, the smaller the block size, then more block loads are required, and the total latency penalty of a small block is relatively larger than that of a large block.

Case2: Full Optimization. As we have seen above, small blocks may degrade the performance of blocked programs. Here we see the coding performance of fully optimized-compressed, fused, and scheduled-version of $P_{\text {enc }}, P_{\text {enc }}^{\text {Full }}$, to check whether or not large blocks better for blocked programs than smaller ones.

\begin{tabular}{|c|c|c|c|c|c|c|c|}
\hline Block size & 64 & 128 & 256 & 512 & $1 \mathrm{~K}$ & $2 \mathrm{~K}$ & $4 \mathrm{~K}$ \\
\hline intel (greedy) & 2.29 & 4.00 & 6.02 & 7.61 & 8.68 & 8.37 & 7.24 \\
\hline intel (dfs) & $-\overline{3} 2$ & $-\overline{-} . \overline{9}$ & 6.09 & $7 . \overline{37}$ & $8.9 \overline{2}$ & $8 . \overline{5} \overline{5}$ & $\overline{7} . \overline{4}$ \\
\hline amd (greedy) & 1.91 & 3.30 & 4.36 & 5.07 & 6.08 & 7.32 & 7.15 \\
\hline$\overline{\mathbf{a m d}}(\overline{\mathrm{df}} \mathrm{s})$ & 1.84 & $3 . \overline{2} 5$ & 4.60 & 5.04 & $6 . \overline{3}$ & $\overline{7.58}$ & $\overline{7} . \overline{31}$ \\
\hline
\end{tabular}

where $\operatorname{NVar}\left(P_{\text {enc }}^{\text {Full }}\right) \sim 90$ and $\operatorname{CCap}\left(P_{\text {enc }}^{\text {Full }}\right) \sim 170$ for all the entries. We should note that our greedy scheduling heuristic generates different programs for each $\mathcal{B}$. However, for all $\mathcal{B}, \mathrm{N} \operatorname{Var}(\cdot)$ is about 90 , and CCap $(\cdot)$ is about 170 . The same is true for the DFS heuristics.

On the basis of the performance, hereafter we set $\mathcal{B}=1 K$ on intel and $\mathcal{B}=2 \mathrm{~K}$ on amd and use the DFS-based scheduling heuristics for comparison with ISA-L and the previous work.

We consider a reason why the scores of $1 K$ and $2 K$ are better than that of $4 K$ in intel. Even if conflicts in cache sets happen, the cache with $1 K$ and $2 K$ blocks may hold more blocks than with $4 K$; therefore, the CPU can efficiently use the cache in the case $1 K$ and $2 K$. On the other hand, in amd, the score of $1 K$ is lower than $2 K$ and $4 K$. It possibly comes from a feature of the microarchitecture, Zen+, of amd's CPU. Zen+, unlike intel's CPU, performs 256 bitwidth instructions of AVX2, splitting it into two 128 bitwidth instructions [38]. To put it simply, the performance for AVX2 of amd is half that of intel. Therefore, in the $1 K$ case of amd, we think that the total latency penalty is more significant than the cache efficiency.

\subsection{Throughput Analysis}

Beyond the average analysis, we optimize the encoding SLP $P_{\text {enc }}$.

\begin{tabular}{|c|c|c|c|c|}
\hline & $P_{\text {enc }}$ & $\mathrm{Co}\left(P_{\text {enc }}\right)$ & $\mathrm{Fu}\left(\mathrm{Co}\left(P_{\mathrm{enc}}\right)\right)$ & $\operatorname{DFs}\left(\mathrm{Fu}\left(\mathrm{Co}\left(P_{\mathrm{enc}}\right)\right)\right)$ \\
\hline$\#_{\oplus}(\cdot)$ & 755 & 385 & 146 & $\leftarrow$ \\
\hline${ }_{M}-\overline{(}(\cdot)$ & $2 \overline{2} 65$ & $11 \overline{5} 5$ & $6 \overline{7} \overline{-}$ & $\longleftarrow$ \\
\hline$\underset{-}{\bar{N}} \overline{\mathrm{V}} \bar{a} \bar{r}$ & $32-$ & 385 & $1 \overline{4} 6$ & 88 \\
\hline$\overline{\mathrm{C}} \overline{\mathrm{C}} \overline{\mathrm{p}}$ & 92 & $4 \overline{47}$ & $22 \overline{-}$ & 167 \\
\hline $\operatorname{intel}(1 K)$ & 4.03 & 4.36 & 7.50 & 8.92 \\
\hline $\operatorname{amd}(2 K)$ & 3.17 & 4.46 & 6.62 & 7.58 \\
\hline
\end{tabular}

where we note that our scheduling heuristics do not affect the number of XORs and memory accesses, and we represent it by $\leftarrow$.

To maximize performance, we can say that compression, fusion, and scheduling are all necessary. Comparing the first and second columns, we tell that the number of memory accesses is more dominant than that of CCap on the performance. On the other hand, comparing the third and fourth columns, we tell that CCap certainly represents the cache efficiency.

We also measure the performance of unoptimized and optimized versions of decoding SLPs. Here, we consider the decoding SLP $P_{\mathrm{dec}}$ obtained by removing $\{2,4,5,6\}$ rows from the encoding matrix

\begin{tabular}{|c|c|c|c|c|c|}
\hline & & $\mathrm{c}$ & $\begin{array}{c}{ }^{\#_{M}} \\
\text { Enc Dec }\end{array}$ & $\begin{array}{c}\text { NVAR } \\
\text { Enc Dec }\end{array}$ & $\begin{array}{c}\text { CCap } \\
\text { Enc Dec }\end{array}$ \\
\hline $\mathbf{R S}(8,4)$ & 121 & 170 & 543747 & $79 \quad 102$ & 143166 \\
\hline $\mathbf{R S}(9,4)$ & 132 & 182 & 611829 & $83 \quad 117$ & 155189 \\
\hline $\mathbf{R S}(10,4)$ & 146 & 206 & $677 \quad 923$ & $88 \quad 125$ & 167205 \\
\hline $\mathbf{R S}(8,3)$ & 75 & 129 & $364 \quad 561$ & $\begin{array}{ll}45 & 77\end{array}$ & 109141 \\
\hline $\mathbf{R S}(9,3)$ & 87 & 144 & $417 \quad 641$ & $58 \quad 91$ & 128163 \\
\hline $\mathbf{R S}(10,3)$ & 96 & 145 & $471 \quad 661$ & $69 \quad 85$ & 148165 \\
\hline $\mathbf{R S}(8,2)$ & 26 & 65 & 180286 & $17 \quad 38$ & $80 \quad 102$ \\
\hline $\mathbf{R S}(9,2)$ & 29 & 73 & 202322 & $19 \quad 42$ & $90 \quad 113$ \\
\hline $\mathbf{R S}(10,2)$ & 30 & 77 & $222 \quad 352$ & $19 \quad 50$ & $98 \quad 130$ \\
\hline
\end{tabular}

Figure 1: Values of $\#_{\oplus}, \#_{M}, \operatorname{NVAR}(\cdot)$, and $\operatorname{CCap}(\cdot)$, of optimized coding SLPs for various codec

because this SLP has the most XORs-1368 as we see in the following table-among decoding SLPs. The following table summarizes the related numbers and decoding performance of $P_{\mathrm{dec}}$ :

\begin{tabular}{|c|c|c|c|c|}
\hline & $P_{\mathrm{dec}}$ & $\mathrm{Co}\left(P_{\mathrm{dec}}\right)$ & $\mathrm{Fu}\left(\mathrm{Co}\left(P_{\mathrm{dec}}\right)\right)$ & $\operatorname{DFs}\left(\operatorname{Fu}\left(\operatorname{Co}\left(P_{\mathrm{dec}}\right)\right)\right)$ \\
\hline$\#_{\oplus}$ & 1368 & 511 & 206 & $\leftarrow$ \\
\hline$\# M$ & 4104 & $15 \overline{3} 3$ & 923 & $\leftarrow$ \\
\hline$-\bar{N} \bar{V} \overline{a r}$ & $-\overline{3}-$ & $\overline{5} \overline{11}$ & $20 \overline{6}$ & $-1 \overline{2} 5^{-}$ \\
\hline$\overline{\mathrm{C}} \overline{\mathrm{C}} \mathrm{ap}$ & 89 & $\overline{585}$ & 283 & 205 \\
\hline $\operatorname{intel}(1 K)$ & 2.35 & 3.32 & 5.51 & 6.67 \\
\hline $\operatorname{amd}(2 K)$ & 2.28 & 3.58 & 5.27 & 6.01 \\
\hline
\end{tabular}

Since $P_{\mathrm{dec}}$ has more instructions than $P_{\mathrm{enc}}$, we can see that the throughputs of $P_{\text {dec }}$ is smaller than those of $P_{\text {enc. }}$ On the other hand, the overall trend is consistent with $P_{\text {enc }}$.

\subsection{Throughput Comparison}

We compare the performance of our fully optimized versions of $P_{\text {enc }}$ and $P_{\text {dec }}$ with ISA-L v2.30.0 [53] and values in [103]. As the same as [103], we consider three kinds of codec; 4-parities $\operatorname{RS}(d, 4)$, 3-parities $\mathbf{R S}(d, 3)$, and 2-parities $\mathbf{R S}(d, 2)$. We summarize main measures for each codec in Figure 1. These values correspond to the rightmost value of the above tables in §7.5.

We compare the coding throughputs of $\mathbf{R S}(d, 4)$ on intel where we use $\mathcal{B}=1 K$ as our blocksize:

\begin{tabular}{c|cc|cc|lc|} 
intel 1K & \multicolumn{2}{|c|}{ Ours } & \multicolumn{2}{|c|}{ ISA-L v2.30 } & \multicolumn{2}{c|}{ Values of [103] } \\
$(\mathrm{GB} / \mathrm{sec})$ & Enc & Dec & Enc & Dec & Enc & Dec \\
\hline RS $(8,4)$ & 8.86 & 6.78 & 7.18 & 7.04 & 4.94 & 4.50 \\
RS $(9,4)$ & 8.83 & 6.71 & 6.91 & 6.58 & \multicolumn{2}{|c|}{ Not Available in [103] } \\
RS(10,4) & 8.92 & 6.67 & 6.79 & 4.88 & 4.94 & 4.71
\end{tabular}

The table claims that our EC library exceeds ISA-L in encoding and parallels in decoding.

Let us consider why there is no difference in the performance between $\mathbf{R S}(10,4)$ and $\mathbf{R S}(8,4)$, although $\mathbf{R S}(8,4)$ is better than $\mathbf{R S}(10,4)$ in terms of the measures in Figure 1 . To encode or decode a given data of $N$-bytes, we run SLPs for $8 \times 8$ input arrays of $\frac{N}{8 \times 8}$ bytes on $\mathbf{R S}(8,4)$. Similarly, we run SLPs for $8 \times 10$ input arrays of $\frac{N}{8 \times 10}$-bytes on $\mathbf{R S}(10,4)$. The difference in Figure 1 is due to the fact that the number of input arrays of $\mathbf{R S}(10,4)$ is larger than that of $\mathbf{R S}(8,4)$. On the other hand, the total number of iterations $\frac{N}{8 \times 8 \times \mathcal{B}}$ on $\mathbf{R S}(8,4)$ is larger than that $\frac{N}{8 \times 10 \times \mathcal{B}}$ on $\mathbf{R S}(10,4)$. As a result, there is no difference in the performance between them.

The encoding and decoding performance of ISA-L are close; however, there is indeed difference between them in our EC library. 
Our encoding and decoding matrices, which are sources of $P_{\mathrm{enc}}$ and $P_{\mathrm{dec}}$, equal those of ISA-L in the binary representation. Namely, we use the same matrix that ISA-L uses. We believe that this situation could be caused by the following fact. In ISA-L or EC libraries based on $\mathrm{MM}$ over finite fields algorithms, for coding matrices $M_{1}$ and $M_{2}$ and a data matrix $D$, there is not much difference between the two computational costs of $M_{1} \cdot D$ and $M_{2} \cdot D$ because finite field multiplication is usually implemented using a multiplication table $\mathcal{M}$; i.e., $a \cdot b$ is computed by accessing $\mathcal{M}[a][b]$. On the other hand, in our libraries or EC libraries based on the method XOR-based EC, even if the sizes of two matrices $M_{1}$ and $M_{2}$ are equal, the number of required XORs could be very different. For example, for an element $e_{1}, e_{2} \in \mathbb{F}_{2^{8}}$, the number of 1 in the bitmatrix $\tilde{e_{1}}$ may be significantly larger than those of $\tilde{e_{2}}$. In a conclusion, we consider the performance gap between encoding and decoding in our library is intrinsic in XOR-based EC.

We also have similar structures in the throughputs table of $\operatorname{RS}(d, 4)$ on amd where we use $\mathcal{B}=2 K$ as the blocksize:

\begin{tabular}{c|cc|cc|lc|} 
amd 2K & \multicolumn{2}{|c|}{ Ours } & \multicolumn{2}{|c|}{ ISA-L v2.30 } & \multicolumn{2}{c|}{ Values of [103] } \\
$(\mathrm{GB} / \mathrm{sec})$ & Enc & Dec & Enc & Dec & Enc & Dec \\
\hline RS $(8,4)$ & 7.09 & 5.53 & 4.60 & 4.61 & 4.69 & 4.06 \\
RS(9,4) & 7.22 & 5.86 & 4.70 & 4.70 & Not Available in [103] \\
RS(10,4) & 7.58 & 6.01 & 4.76 & 4.75 & 4.67 & 3.91
\end{tabular}

Comparison in Low Parities. We compare $\mathbf{R S}(d, 3)$ and $\mathbf{R S}(d, 2)$ :

\begin{tabular}{|c|c|c|c|c|c|c|}
\hline \multirow{2}{*}{$\begin{array}{c}\text { intel 1K } \\
(\mathrm{GB} / \mathrm{sec})\end{array}$} & \multicolumn{2}{|c|}{ Ours } & \multicolumn{2}{|c|}{ ISA-L v 2.30} & \multicolumn{2}{|c|}{ Values of [103] } \\
\hline & Enc & Dec & Enc & Dec & Enc & Dec \\
\hline $\mathbf{R S}(8,3)$ & 12.32 & 8.82 & 9.09 & 9.25 & 6.08 & 5.57 \\
\hline $\mathbf{R S}(9,3)$ & 11.97 & 8.27 & 7.31 & 7.92 & 6.17 & 5.66 \\
\hline $\mathbf{R S}(10,3)$ & 11.78 & 8.89 & 6.78 & 7.93 & $6.15_{S}$ & 5.90 \\
\hline $\mathbf{R S}(8,2)$ & 18.79 & 14.59 & 12.99 & 13.34 & $8.13_{E}$ & $8.07_{E}$ \\
\hline $\mathbf{R S}(9,2)$ & 18.93 & 14.27 & 11.85 & 12.03 & $8.34_{E}$ & 8.04 \\
\hline $\mathbf{R S}(10,2)$ & 18.98 & 14.66 & 12.12 & 12.61 & $8.40_{E}$ & $8.22_{E}$ \\
\hline amd $2 K$ & \multicolumn{2}{|c|}{ Ours } & \multicolumn{2}{|c|}{ ISA-L v 2.30} & \multicolumn{2}{|c|}{ Values of [103] } \\
\hline$(\mathrm{GB} / \mathrm{sec})$ & Enc & Dec & Enc & Dec & Enc & Dec \\
\hline $\mathbf{R S}(8,3)$ & 9.35 & 7.43 & 5.01 & 4.93 & $6.38_{S}$ & $5.18_{Q}$ \\
\hline $\mathbf{R S}(9,3)$ & 9.41 & 7.44 & 5.07 & 5.02 & $6.53_{S}$ & $6.53 \hat{S}$ \\
\hline $\mathbf{R S}(10,3)$ & 9.51 & 7.46 & 5.04 & 5.02 & $6.49_{S}$ & ${ }^{5.31_{Q}}$ \\
\hline $\mathbf{R S}(8,2)$ & 13.60 & 12.07 & 7.11 & 7.09 & $8.96_{R}$ & $10.11_{E}$ \\
\hline $\mathbf{R S}(9,2)$ & 13.83 & 12.06 & 7.17 & 7.19 & $9.12_{R}$ & $9.31_{R}$ \\
\hline $\mathbf{R S}(10,2)$ & 14.13 & 12.19 & 7.24 & 7.15 & $9.31_{R}$ & $10.60_{R}$ \\
\hline
\end{tabular}

The column "Values of [103]" consists of the best throughputs among results of the corresponding parameters in [103] where the authors compared their proposal method with some codecs specialized for low parities-STAR [51] and QFS [77] for three parities, and EvenOdd [12] and RDP [31] for two parities. Indeed, the values $\cdot S, \cdot Q, \cdot E$, and $\cdot_{R}$ are scored by STAR, QFS, EvenOdd, and $\mathrm{RDP}$, respectively (the other values are scored by their proposal approach). We can say our library works well without specializing for low parities.

\section{CONCLUSION AND FUTURE WORK}

We have proposed a streamlined approach to implement an efficient XOR-based EC library. We combined the four notions, straight-line programs (SLPs) from program optimization, grammar compression algorithm REPAIR, the functional program optimization technique deforestation, and the pebble game from program analysis. We extended RePaIr to our XorRePAIR to accommodate the cancellative property of XOR. We used the pebble game to model SLPs with the abstract LRU cache. Orthogonally composing these methods, we have implemented an experimental library that outperforms Intel's high-performance library, ISA-L [52].

Analyzing the result of experiments, we have noticed the importance of cache optimization. In this paper, we only tried to abstract the L1 cache but not the L2 and L3 caches. We are thinking about using the multilevel pebble game introduced by Savage in [88] to accommodate the L2 and L3. As a related cache efficiency topic, we are interested in automatically inserting software prefetches [68]. It may hide the cache transfer penalty from memory to cache if a CPU concentrates on performing array XORs against cached data.

\section{ACKNOWLEDGMENTS}

We gratefully thank anonymous reviewers for their invaluable and thorough comments, which improved the presentation of this paper and also helped us improve the performance of our experimental library. Many thanks to our colleague Masahiro Fukasawa for fruitful discussions of cache optimization. Thanks also to Iori Yoneji for his full support in providing evaluation environments.

\section{REFERENCES}

[1] A. V. Aho, S. C. Johnson, and J. D. Ullman. 1977. Code Generation for Expressions with Common Subexpressions. F. ACM 24, 1 (1977), 146-160.

[2] A. V. Aho, R. Sethi, and J. D. Ullman. 1986. Compilers: Principles, Techniques, and Tools. Addison-Wesley.

[3] A. V. Aho and J. D. Ullman. 1972. Optimization of Straight Line Programs. SIAM f. Comput. 1, 1 (1972), 1-19.

[4] F. E. Allen and J. Cocke. 1972. A Catalogue of Optimizing Transformations. In Design and Optimization of Compilers, R. Rustin (Ed.). Prentice-Hall, 1-30.

[5] J. Alman and V. V. Williams. 2021. A Refined Laser Method and Faster Matrix Multiplication. In SODA '21. 522-539.

[6] B. Alpern, M. N. Wegman, and F. K. Zadeck. 1988. Detecting Equality of Variables in Programs. In POPL '88. ACM, 1-11.

[7] AMD. 2020. AMD64 Architecture Programmer's Manual. https://www.amd.com/ system/files/TechDocs/26568.pdf

[8] Apache Hadoop. 2020. HDFS Erasure Coding. https://hadoop.apache.org/docs/ current/hadoop-project-dist/hadoop-hdfs/HDFSErasureCoding.html

[9] A. W. Appel and L. George. 2001. Optimal Spilling for CISC Machines with Few Registers. In PLDI '01. ACM, 243-253.

[10] ARM. 2020. SIMD Neon. https://developer.arm.com/documentation/ddi0487/ latest

[11] Storage Networking Industry Association. 2009. Common RAID Disk Data Format. https://www.snia.org/tech_activities/standards/curr_standards/ddf

[12] M. Blaum, J. Brady, J. Bruck, and Jai Menon. 1995. EVENODD: an efficient scheme for tolerating double disk failures in RAID architectures. IEEE Trans. Comput. 44, 2 (1995), 192-202.

[13] J. Blömer, M. Kalfane, R. Karp, M. Karpinski, M. Luby, and D. Zuckerman. 1995. An XOR-Based Erasure-Resilient Coding Scheme. ICSI Technical Report No. TR-95-048 (1995).

[14] F. Bouchez, A. Darte, C. Guillon, and F. Rastello. 2007. Register Allocation: What Does the NP-Completeness Proof of Chaitin et al. Really Prove? Or Revisiting Register Allocation: Why and How. In LCPC '07. Springer, 283-298.

[15] F. Bouchez, A. Darte, and F. Rastello. 2007. On the Complexity of Register Coalescing. In CGO '07. 102-114.

[16] F. Bouchez, A. Darte, and F. Rastello. 2007. On the Complexity of Spill Everywhere under SSA Form. In LCTES '07. ACM, 103-112.

[17] J. Boyar, P. Matthews, and R. Peralta. 2008. On the Shortest Linear Straight-Line Program for Computing Linear Forms. In MFCS '08. Springer, 168-179.

[18] J. Boyar, P. Matthews, and R. Peralta. 2013. Logic Minimization Techniques with Applications to Cryptology. Journal of Cryptology 26 (2013), 280-312. Issue 2.

[19] M. A. Breuer. 1969. Generation of Optimal Code for Expressions via Factorization. Commun. ACM 12, 6 (June 1969), 333-340.

[20] P. Briggs, K. D. Cooper, and L. Torczon. 1994. Improvements to Graph Coloring Register Allocation. ACM Trans. Program. Lang. Syst. 16, 3 (May 1994), 428-455.

[21] J. Bruno and R. Sethi. 1976. Code Generation for a One-Register Machine. $f$. ACM 23, 3 (1976), 502-510. 
[22] R. M. Burstall and J. Darlington. 1977. A Transformation System for Developing Recursive Programs. F. ACM 24, 1 (1977), 44-67.

[23] T. Carpenter, F. Rastello, P. Sadayappan, and A. Sidiropoulos. 2016. Brief An nouncement: Approximating the I/O Complexity of One-Shot Red-Blue Pebbling In SPAA '16. ACM, 161-163.

[24] Ceph. 2016. Ceph Erasure Code. https://docs.ceph.com/en/latest/rados/ operations/erasure-code/

[25] G. J. Chaitin. 1982. Register Allocation \& Spilling via Graph Coloring. (1982), 98-105.

[26] G. J. Chaitin, M. A. Auslander, A. K. Chandra, J. Cocke, M. E. Hopkins, and P. W Markstein. 1981. Register allocation via coloring. Computer Languages 6, 1 (1981), 47-57.

[27] M. Charikar, E. Lehman, D. Liu, R. Panigrahy, M. Prabhakaran, A. Sahai, and A. Shelat. 2005. The smallest grammar problem. IEEE Trans. on Information Theory 51, 7 (2005), 2554-2576.

[28] S. Coleman and K. S. McKinley. 1995. Tile Size Selection Using Cache Organization and Data Layout. In PLDI '95. ACM, 279-290.

[29] J. Cook, R. Primmer, and A. de Kwant. 2013. Comparing cost and performance of replication and erasure coding. CoRR abs/1308.1887 (2013). http://arxiv.org/ abs/1308.1887

[30] S. A. Cook. 1971. The Complexity of Theorem-Proving Procedures. In STOC '71. ACM, 151-158.

[31] P. Corbett, B. English, A. Goel, T. Grcanac, S. Kleiman, J. Leong, and S. Sankar. 2004. Row-Diagonal Parity for Double Disk Failure Correction. In FAST '04. USENIX Association.

[32] D. Coutts, R. Leshchinskiy, and D. Stewart. 2007. Stream Fusion: From Lists to Streams to Nothing at All. In ICFP '07. ACM, 315-326.

[33] R. Cytron, J. Ferrante, B. K. Rosen, M. N. Wegman, and F. K. Zadeck. 1991. Efficiently Computing Static Single Assignment Form and the Control Dependence Graph. Trans. on Programming Languages and Systems 13, 4 (Oct 1991), 451-490.

[34] E. D. Demaine and Q. C. Liu. 2017. Inapproximability of the Standard Pebble Game and Hard to Pebble Graphs. In WADS '07. Springer, 313-324.

[35] E. D. Demaine and Q. C. Liu. 2018. Red-Blue Pebble Game: Complexity of Computing the Trade-Off between Cache Size and Memory Transfers. In SPAA '18. ACM, 195-204

[36] A. P. Ershov. 1958. On Programming of Arithmetic Operations. Commun. ACM 1, 8 (Aug. 1958), 3-6.

[37] M. Farach-Colton and V. Liberatore. 2000. On Local Register Allocation. Journal of Algorithms 37, 1 (2000), 37-65.

[38] A. Fog. 2021. The microarchitecture of Intel, AMD, and VIA CPUs. https://www. agner.org/optimize/microarchitecture.pdf

[39] M. R. Garey and D. S. Johnson. 1979. Computers and Intractability; A Guide to the Theory of NP-Completeness. W. H. Freeman \& Co.

[40] L. George and A. W. Appel. 1996. Iterated Register Coalescing. ACM Trans. Program. Lang. Syst. 18, 3 (May 1996), 300-324.

[41] A. Gill, J. Launchbury, and S. L. P. Jones. 1993. A Short Cut to Deforestation. In FPCA' '93. ACM, 223-232.

[42] A. González, F. Latorre, and G. Magklis. 2010. Processor Microarchitecture: An Implementation Perspective. Morgan \& Claypool Publishers.

[43] D. Grund and S. Hack. 2007. A Fast Cutting-Plane Algorithm for Optimal Coalescing. In CC '07. Springer, 111-125.

[44] S. Hack, D. Grund, and G. Goos. 2006. Register Allocation for Programs in SSA-Form. In CC '06. Springer, 247-262.

[45] P. Hammarlund, A. J. Martinez, A. A. Bajwa, D. L. Hill, E. Hallnor, H. Jiang, M Dixon, M. Derr, M. Hunsaker, R. Kumar, R. B. Osborne, R. Rajwar, R. Singhal, R. D'Sa, R. Chappell, S. Kaushik, S. Chennupaty, S. Jourdan, S. Gunther, T. Piazza, and T. Burton. 2014. Haswell: The Fourth-Generation Intel Core Processor. IEEE Micro 34, 2 (2014), 6-20.

[46] J. L. Hennessy and D. A. Patterson. 2017. Computer Architecture, Sixth Edition: A Quantitative Approach (6th ed.). Morgan Kaufmann Publishers Inc.

[47] J-W. Hong and H. T. Kung. 1981. I/O Complexity: The Red-Blue Pebble Game. In STOC ' 81 . ACM, 326-333.

[48] C. Huang, J. Li, and M. Chen. 2007. On Optimizing XOR-Based Codes for Fault-Tolerant Storage Applications. In ITW'07. 218-223.

[49] C. Huang, H. Simitci, Y. Xu, A. Ogus, B. Calder, P. Gopalan, J. Li, and S. Yekhanin 2012. Erasure Coding in Windows Azure Storage. In USENIX ATC'12. USENIX.

[50] C. Huang and L. Xu. 2003. Fast software implementation of finite field operations. Technical Report. Washington University.

[51] C. Huang and L. Xu. 2008. STAR : An Efficient Coding Scheme for Correcting Triple Storage Node Failures. IEEE Trans. Comput. 57, 7 (2008), 889-901.

[52] Intel. [n.d.]. Intelligent Storage Acceleration Library. https://github.com/intel/isa1/

[53] Intel. [n.d.]. Intelligent Storage Acceleration Library (version 2.30.0). https: //github.com/intel/isa-1/releases/tag/v2.30.0

[54] Intel. 2017. ISA-L performance report. https://01.org/intel\%C2\%AE-storageacceleration-library-open-source-version/documentation/documentation

[55] Intel. 2020. Intel 64 and IA-32 architectures optimization reference manual. https: //software.intel.com/content/www/us/en/develop/articles/intel-sdm.html
[56] Intel. 2021. Intel Architecture Instruction Set Extensions Programming Reference. https://software.intel.com/content/www/us/en/develop/download/intelarchitecture-instruction-set-extensions-programming-reference.html

[57] S. Kalcher and V. Lindenstruth. 2011. Accelerating Galois Field Arithmetic for Reed-Solomon Erasure Codes in Storage Applications. In CLUSTER '11. 290-298.

[58] Richard M. Karp. 1972. Reducibility among Combinatorial Problems. Springer, 85-103.

[59] C. K. Koc and T. Acar. 1998. Montgomery Multiplication in GF(2k). Designs, Codes and Cryptography 14, 1 (1998), 57-69.

[60] T. Kranz, G. Leander, K. Stoffelen, and F. Wiemer. 2017. Shorter Linear StraightLine Programs for MDS Matrices. IACR Trans. on Symmetric Cryptology 2017, 4 (2017), 188-211.

[61] G. Kwasniewski, M. Kabić, M. Besta, J. VandeVondele, R. Solcà, and T. Hoefler. 2019. Red-Blue Pebbling Revisited: Near Optimal Parallel Matrix-Matrix Multiplication. In SC' 19. ACM, Article 24.

[62] F. L. Gall. 2014. Powers of Tensors and Fast Matrix Multiplication. In ISSAC '14. ACM, 296-303.

[63] J. Lacan and J. Fimes. 2004. Systematic MDS erasure codes based on Vandermonde matrices. IEEE Communications Letters 8, 9 (2004), 570-572.

[64] M. D. Lam, E. E. Rothberg, and M. E. Wolf. 1991. The Cache Performance and Optimizations of Blocked Algorithms. In ASPLOS '91. ACM, 63-74.

[65] S. Lang. 1986. Introduction to Linear Algebra. Springer.

[66] R. Larrieu. 2019. Fast finite field arithmetic. Ph.D. Dissertation. University of Paris-Saclay, France.

[67] N. J. Larsson and A. Moffat. 1999. Offline dictionary-based compression. In DCC'99. 296-305.

[68] J. Lee, H. Kim, and R. Vuduc. 2012. When Prefetching Works, When It Doesn't, and Why. ACM Trans. Archit. Code Optim. 9, 1, Article 2 (March 2012).

[69] T. Lengauer and R. E. Tarjan. 1980. The space complexity of pebble games on trees. Inform. Process. Lett. 10, 4 (1980), 184-188.

[70] S. Ling and C. Xing. 2004. Coding Theory: A First Course. Cambridge University Press.

[71] J. W. H. Liu. 1986. On the Storage Requirement in the Out-of-Core Multifrontal Method for Sparse Factorization. ACM Trans. Math. Softw. 12, 3 (Sept. 1986), 249-264.

[72] J. Luo, M. Shrestha, L. Xu, and J. S. Plank. 2014. Efficient Encoding Schedules for XOR-Based Erasure Codes. IEEE Trans. Comput. 63, 09 (2014), 2259-2272.

[73] F. MacWilliams and N. Sloane. 1977. The Theory of Error-Correcting Codes. Elsevier.

[74] E. D. Mastrovito. 1989. VLSI designs for multiplication over finite fields GF(2m). In Applied Algebra, Algebraic Algorithms and Error-Correcting Codes. Springer, 297-309.

[75] S. Muchnick. 1998. Advanced Compiler Design and Implementation. Morgan Kaufmann Publishers Inc.

[76] I. Nakata. 1967. On Compiling Algorithms for Arithmetic Expressions. Commun. ACM 10, 8 (Aug. 1967), 492-494.

[77] M. Ovsiannikov, S. Rus, D. Reeves, P. Sutter, S. Rao, and J. Kelly. 2013. The Quantcast File System. VLDB Endow. 6, 11 (Aug. 2013), 1092-1101.

[78] C. Paar. 1997. Optimized arithmetic for Reed-Solomon encoders. In IEEE Intern. Symp. on Information Theory. 250-250.

[79] P.R. Panda, H. Nakamura, N.D. Dutt, and A. Nicolau. 1999. Augmenting loop tiling with data alignment for improved cache performance. IEEE Trans. Comput. 48, 2 (1999), 142-149.

[80] P. A. Papp and R. Wattenhofer. 2020. On the Hardness of Red-Blue Pebble Games. In SPAA '20. ACM, 419-429.

[81] F. M. Q. Pereira and J. Palsberg. 2005. Register Allocation Via Coloring of Chordal Graphs. In APLAS '05. Springer, 315-329.

[82] J. Plank. 2008. The RAID-6 liberation codes. In FAST '08. 97-110.

[83] J. Plank, K. Greenan, and E. L. Miller. 2013. Screaming Fast Galois Field Arithmetic Using Intel SIMD Extensions. In FAST'13.

[84] I. S. Reed and G. Solomon. 1960. Polynomial Codes Over Certain Finite Fields. 7. Soc. Indust. Appl. Math. 8, 2 (1960), 300-304.

[85] A. Reyhani-Masoleh, M. Taha, and D. Ashmawy. 2018. Smashing the Implementation Records of AES S-box. IACR Trans. on Cryptographic Hardware and Embedded Systems 2018, 2 (2018), 298-336.

[86] G. Rivera and C.-W. Tseng. 1999. A Comparison of Compiler Tiling Algorithms. In $C C^{\prime} 99$. Springer, 168-182.

[87] B. K. Rosen, M. N. Wegman, and F. K. Zadeck. 1988. Global Value Numbers and Redundant Computations. In POPL '88. ACM, 12-27.

[88] J. E. Savage. 1995. Extending the Hong-Kung Model to Memory Hierarchies. In COCOON'95. Springer, 270-281.

[89] V. Schneider. 1971. On the number of registers needed to evaluate arithmetic expressions. BIT Numerical Mathematics 11, 1 (01 Mar 1971), 84-93.

[90] R. Sethi. 1973. Complete Register Allocation Problems. In STOC '73. ACM, 182-195.

[91] R. Sethi. 1975. Complete Register Allocation Problems. SIAM f. Comput. 4, 3 (1975), 226-248. 
[92] R. Sethi and J. D. Ullman. 1970. The Generation of Optimal Code for Arithmetic Expressions. F. ACM 17, 4 (Oct. 1970), 715-728.

[93] A. Shenoy. 2015. The Pros and Cons of Developing Erasure Coding and Replication Instead of Traditional RAID in Next-Generation Storage Platforms. (2015). https://www.snia.org/educational-library/pros-and-cons-developingerasure-coding-and-replication-instead-traditional-raid SDC '15.

[94] G.E. Shilov. 1977. Linear Algebra. Dover Publications, Inc.

[95] K. Stoffelen. 2016. Optimizing S-Box Implementations for Several Criteria Using SAT Solvers. In FSE '16. Springer, 140-160.

[96] A. Takano and E. Meijer. 1995. Shortcut Deforestation in Calculational Form. In FPCA '95. ACM, 306-313.

[97] Q. Q. Tan and T. Peyrin. 2019. Improved Heuristics for Short Linear Programs. IACR Trans. on Cryptographic Hardware and Embedded Systems 2020, 1 (2019), 203-230.

[98] Y. Uezato. 2021. Author's Github Repository. https:/github.com/yuezato/xorslp_ ec

[99] P. Wadler. 1989. Theorems for Free!. In FPCA '89. ACM, 347-359.

[100] P. Wadler. 1990. Deforestation: transforming programs to eliminate trees. Theoretical Computer Science 73, 2 (1990), 231-248.

[101] H. Weatherspoon and J. Kubiatowicz. 2002. Erasure Coding Vs. Replication: A Quantitative Comparison. In IPTPS '01. Springer, 328-338.

[102] K. Yotov, X. Li, G. Ren, M. Cibulskis, G. DeJong, M. Garzaran, D. Padua, K. Pingali, P. Stodghill, and P. Wu. 2003. A Comparison of Empirical and Model-Driven Optimization. In PLDI '03. ACM, 63-76.

[103] T. Zhou and C. Tian. 2020. Fast Erasure Coding for Data Storage: A Comprehensive Study of the Acceleration Techniques. ACM Trans. Storage 16, 1, Article 7 (2020).

\section{A PROOF OF THEOREM 1}

In this appendix section, we show the following problem of $\S 5$ is NP-complete.

\section{The minimum memory access problem}

For an $P \in \mathbb{S L P}_{\oplus}$, we find $Q \in \mathbb{S L P}_{\vec{\oplus}}$ that satisfies $\llbracket P \rrbracket=\llbracket Q \rrbracket$ and minimizes ${ }_{M}(Q)$.

\section{A.1 Vertex Cover Problem}

To show the NP-completeness of our problem, we use the classic NP-complete Vertex Cover problem [39].

The vertex cover problem (VCP) is a decision problem such that:

- Let $G$ be an undirected graph $G$ and $k$ be a natural number.

- We then decide if there is a node set $X$ of $G$ such that

$-|X| \leq k ;$ and

- $G$ is covered by $X$. Namely, every edge $(a, b)$ of $G$ is covered by $X$; i.e., $a \in X$ or $b \in X$ holds.

The optimization version of VCP, OptVCP, is an optimization problem such that:

- Let $G$ be an undirected graph.

- We then compute the smallest node set $X$ of $G$ that covers $G$.

Since VCP is NP-complete, OptVCP cannot be solved in polynomial time unless $\mathbf{P}=\mathbf{N P}$.

Below we reduce our optimization problem, the minimum memory access problem, to OptVCP.

\section{A.2 Build SLP from Graph}

Let $G$ be an undirected graph.

Before building an SLP corresponding to $G$, we modify $G$ for our construction and proof as follows:

- For each node $a$ of $G$, we add two fresh nodes $\lambda_{a}$ and $\mu_{a}$ and add two edges $\left(a, \lambda_{a}\right)$ and $\left(a, \mu_{a}\right)$.

- We call added nodes $\lambda_{\bullet}$ and $\mu_{\bullet}$ local nodes.
- For a node $a$ of $G$, we call edges $\left(a, \lambda_{a}\right)$ and $\left(a, \mu_{a}\right)$ local edges.

Hereafter we omit the subscripts of $\lambda_{\bullet}$ and $\mu_{\bullet}$ if they are clear from the context. For example, we can simply write $(a, \lambda)$ because there is no edge such that $\left(b, \lambda_{a}\right)$.

For the modified graph, we build an SLP as follows:

- For each edge $(x, y) \in G_{\text {modif }}$, we add a goal $g_{x y} \leftarrow \rho \oplus x \oplus y$. Let us consider the following example:
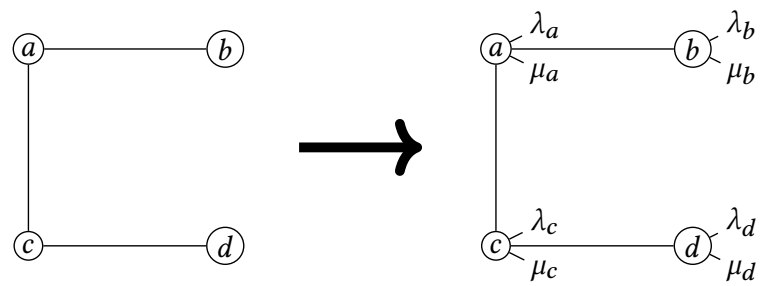

The left graph $G$ is modified to the right graph $G_{\text {modif. }}$ We build the SLP $P_{G}$ from $G_{\text {modif }}$ as follows:

$$
\begin{aligned}
& g_{a b} \leftarrow \rho \oplus a \oplus b \\
& g_{a c} \leftarrow \rho \oplus a \oplus c \\
& g_{c d} \leftarrow \rho \oplus c \oplus d \\
& g_{a \lambda_{a}} \leftarrow \rho \oplus a \oplus \lambda_{a} \\
& g_{a \mu_{a}} \leftarrow \rho \oplus a \oplus \mu_{a} ; \\
& \vdots \\
& g_{d \lambda_{d}} \leftarrow \rho \oplus a \oplus \lambda_{d} \\
& g_{d \mu_{d}} \leftarrow \rho \oplus a \oplus \mu_{d} \\
& \operatorname{ret}\left(g_{a b}, g_{a c}, g_{c d}, g_{a \lambda_{a}}, \ldots, g_{d \mu_{d}}\right)
\end{aligned}
$$

We will show that we can extract the minimum cover sets of $G$ (rather than $G_{\text {modif }}$ ) from the solution of the minimum memory access problem for $P_{G}$. For example, the following $\mathbb{S L I}_{\vec{\oplus}}$ is one of the minimum solution:

$$
\begin{aligned}
& \Gamma_{a} \leftarrow \rho \oplus a ; \\
& g_{a b} \leftarrow \Gamma_{a} \oplus b ; \quad g_{a c} \leftarrow \Gamma_{a} \oplus c ; \quad g_{a \lambda} \leftarrow \Gamma_{a} \oplus \lambda ; \quad g_{a \mu} \leftarrow \Gamma_{a} \oplus \mu ; \\
& g_{b \lambda} \leftarrow \rho \oplus b \oplus \lambda ; \quad g_{b \mu} \leftarrow \rho \oplus b \oplus \mu ; \\
& \Gamma_{c} \leftarrow \rho \oplus c ; \\
& g_{c d} \leftarrow \Gamma_{c} \oplus d ; \quad g_{c \lambda} \leftarrow \Gamma_{c} \oplus \lambda ; \quad g_{c \mu} \leftarrow \Gamma_{c} \oplus \mu ; \\
& g_{d \lambda} \leftarrow \rho \oplus d \oplus \lambda ; \quad g_{d \mu} \leftarrow \rho \oplus d \oplus \mu ; \\
& \operatorname{ret}(\cdots)
\end{aligned}
$$

From the solution, we extract $a$ and $c$ as cover sets of the original graph $G$; actually, $\{a, c\}$ covers $G$.

Technically, we prove the following two lemmas in the subsequent sections.

Lemma A1. Let $Q$ be an $\mathbb{S L P} \vec{\oplus}$ where $\llbracket P_{G} \rrbracket=\llbracket Q \rrbracket$. We can effectively normalize $Q$ to $Q^{\prime}$ such that;

- $\#_{M}\left(Q^{\prime}\right) \leq \#_{M}(Q)$.

- Every edge $(a, b)$ of $G$ is represented in $Q^{\prime}$ as follows:

$$
\begin{aligned}
& \Gamma_{a} \leftarrow \rho \oplus a ; \\
& g_{a b} \leftarrow \Gamma_{a} \oplus b
\end{aligned}
$$


- For a node a of $G$, the local edges $(a, \lambda)$ and $(a, \mu)$ are represented in $Q^{\prime}$ as follows:

$$
\left(\begin{array}{l}
\text { if } \Gamma_{a} \text { is in } Q^{\prime} \\
\hline g_{a \lambda} \leftarrow \Gamma_{a} \oplus \lambda ; \\
g_{a \mu} \leftarrow \Gamma_{a} \oplus \mu .
\end{array}\right) \quad \text { OR } \quad\left(\begin{array}{l}
\text { if } \Gamma_{a} \text { is not in } Q^{\prime} \\
\hline g_{a \lambda} \leftarrow \rho \oplus a \oplus \lambda ; \\
g_{a \mu} \leftarrow \rho \oplus a \oplus \mu .
\end{array}\right)
$$

Using this lemma, we obtain the following two useful properties.

Lemma A2. Let $P$ be a normalized $\mathbb{S L P} \mathbb{P}_{\vec{\oplus}}$ in the meaning of Lemma A1. If the size of the set $\left\{a \in G: \Gamma_{a}\right.$ is in $\left.Q^{\prime}\right\}$ is $k$, then

$$
3|E|+8|N|+k=\#_{M}\left(Q^{\prime}\right) .
$$

where $E$ is the edge sets of $G$ and $N$ is the node sets of $G$.

Proof. For each temporal variables, we need 3-costs for defining $\Gamma_{a} \leftarrow \rho \oplus a$. This totally require $3 k$-costs.

For each local edge of a node $a$,

- if we have $\Gamma_{a}$, we require 6-costs: $g_{a \lambda} \leftarrow \Gamma_{a} \oplus \lambda$ and $g_{a \mu} \leftarrow$ $\Gamma_{a} \oplus \mu$.

- Otherwise, we require 8-costs: $g_{a \lambda} \leftarrow \rho \oplus a \oplus \lambda$ and $g_{a \mu} \leftarrow$ $\rho \oplus a \oplus \mu$.

Totally, for defining all the local edges, we require $6 k+8(|N|-k)$ costs.

For each edge $(a, b)$ of $G$, since we have $\Gamma_{a}$ or $\Gamma_{b}$, we require 3-costs $g_{a b} \leftarrow \Gamma_{a} \oplus b$ or $g_{a b} \leftarrow \Gamma_{b} \oplus a$.

Entirely, we need the following costs matching with one of the statement:

$$
3 k+(6 k+8(|N|-k))+3|E|=k+8|N|+3|E| .
$$

Using these lemmas, we obtain the following property.

Lemma A3. For a given graph $G$, let $Q$ be the ${ }_{M}$-minimum SLP such that $\llbracket Q \rrbracket=\llbracket P_{G} \rrbracket$. The following holds on $Q$ :

- There is a number $k$ such that $3|E|+8|N|+k=\#_{M}(Q)$.

- Furthermore, $k$ is the size of the smallest cover set of $G$.

Proof. We normalize $Q$ to $Q^{\prime}$ using Lemma A1 and then estimate $k$ using Lemma A2. The conditions of Lemma A1 tells we can cover the graph $G$ using $k$ vertices.

Let $X=\left\{x_{1}, x_{2}, \ldots, x_{n}\right\}$ be the smallest cover set of $G$. We then can construct an $\mathbb{S L P}_{\vec{\oplus}} R$ such that $R$ satisfies the conditions of Lemma $\mathrm{A} 1$ and $\llbracket R \rrbracket=\llbracket P_{G} \rrbracket$ defining variables $\Gamma_{x_{1}}, \ldots, \Gamma_{x_{n}}$ as

$$
\Gamma_{x_{1}} \leftarrow \rho \oplus x_{1} ; \Gamma_{x_{2}} \leftarrow \rho \oplus x_{2} ; \quad \ldots \quad \Gamma_{x_{n}} \leftarrow \rho \oplus x_{n} ;
$$

Lemma A2 tells $\#_{M}(R)=3|E|+8|N|+n$.

From the $\#_{M}$-minimality of $Q, k \leq n$ must hold. On the other hand, from the minimality of $n, n \leq k$ must hold; then, we have $k=n$.

Lemma A3 immediately leads to the intractability of our optimization problem.

Hereafter, we will show Lemma A1.

\section{A.3 Proof of Lemma A1}

Terminology. The following is terminology for this section:

Temporal variable: If a variable is not a goal, we call it temporal variable.

Goal variable: If a variable is one of the goal, we call it goal variable.

- In our setting, each goal variable is of the form $g_{a b}, g_{a \lambda}$, or $g_{a \mu}$ where $a$ and $b$ are nodes and $\lambda$ and $\mu$ are local nodes.

Freely appearing: If a variable $v$ or a constant $c$ appears in the definition of a temporal variable, we say that $v$ or $c$ freely appears in the program

\section{Metavariable Naming Rule.}

$\boldsymbol{a}, \boldsymbol{b}, \ldots .:$ Metavariables for nodes of $G$.

$\boldsymbol{x}, \boldsymbol{y}, \boldsymbol{z}$ : Metavariables for nodes of $G_{\text {modif }}$.

$t, t_{1}, t_{2}, \ldots:$ Metavariables for terms (i.e., constants or variables) of SLPs.

$v, v_{1}, v_{2}, \ldots:$ Metavariables for a temporal variable of SLPs.

Notation. Let $P$ be an SLP and $v$ be a variable of $P$.

We write $(v)$ to denote the value of $v$. To justify this notation, we assume that every SLP of this section is of the SSA (single static assignment) form where each variable is assigned exactly once [6, 87]. We can easily convert a given SLP to an SSA form SLP without changing the semantics and the size. For example,

\begin{tabular}{lll}
$\frac{\text { non-SSA SLP }}{v \leftarrow a \oplus b \oplus c ;}$ & & $\frac{1}{\text { SSA SLP }}$ \\
\hline$v \leftarrow v \oplus d \oplus e ;$ & & $v_{1} \leftarrow a \oplus b \oplus c ;$ \\
$v \leftarrow v \oplus f ;$ & & $v_{2} \leftarrow v_{1} \oplus d \oplus e ;$ \\
$v \leftarrow v_{2} \oplus f ;$
\end{tabular}

A.3.1 Normalization I.

Let $v$ be a temporal variable.

If $|v|=\{x, y\}$ and $v \leftarrow t_{1} \oplus t_{2} \oplus \cdots$, we convert the definition to the trivial form as follows:

$$
v \leftarrow t_{1} \oplus t_{2} \oplus \cdots ; \Leftrightarrow v \leftarrow x \oplus y ;
$$

If $\| v \mid=\{x, y, z\}$ and $v \leftarrow t_{1} \oplus t_{2} \oplus t_{3} \oplus \cdots$, we convert the definition to the trivial form as follows:

$$
v \leftarrow t_{1} \oplus t_{2} \oplus t_{3} \oplus \cdots ; \Leftrightarrow v \leftarrow x \oplus y \oplus z ;
$$

These conversion do not change the semantics of the program and increase the size of the program.

\section{A.3.2 Normalization II.}

If the program normalized by Normalization-I has a variable $\Gamma_{a}$ of the form:

$$
\Gamma_{a} \leftarrow \rho \oplus a ;
$$

using $\Gamma_{a}$, we rewrite the definitions of $g_{a \lambda}$ and $g_{a \mu}$ as follows:

$$
g_{a \lambda} \leftarrow \Gamma_{a} \oplus \lambda ; \quad g_{a \mu} \leftarrow \Gamma_{a} \oplus \mu ;
$$

The following holds after these normalization steps.

Proposition A1. If $\Gamma_{a}$ is not in the (normalized) program, for $\lambda_{a}$ (also $\mu_{a}$ ), either one of the following holds:

- $g_{a \lambda}$ is defined as $g_{a \lambda} \leftarrow \rho \oplus a \oplus \lambda$; or

- $\lambda$ freely appears.

Proof. We assume that the former does not hold and then consider the following subcases about $g_{a \lambda}$ : 
$g_{a \lambda} \leftarrow v \oplus \lambda$ where $|v|=\{\rho, a\}$ : This contradicts to the assumption.

$g_{a \lambda} \leftarrow v \oplus x\left(x \neq \lambda_{a}\right)$ or $g_{a \lambda} \leftarrow v \oplus \rho$ where $\left.|v|\right)=\{\lambda, \ldots\}:$ To define $v, \lambda$ must freely appear because any other goal does not have $\lambda_{a}$.

$g_{a \lambda} \leftarrow v \oplus g_{x y}$ where $(v)=\{\lambda, \ldots\}:$ To define $v, \lambda$ must freely appear.

$g_{a \lambda} \leftarrow v_{1} \oplus v_{2}$ where $\mid v_{1} D=\{\lambda, \ldots\}$ : To define $v_{1}, \lambda$ must freely appear.

On the basis of this lemma, we introduce one notation.

Movable occurrence: For a local node $\lambda$, if its appears in $g_{a \lambda} \leftarrow \rho \oplus a \oplus \lambda$ or $\lambda$ freely appears as $v \leftarrow \lambda \oplus \cdots$, we call such occurrences of $\lambda$ movable occurrences.

The meaning of the term movable will be justified below.

\section{A.3.3 Normalization III: Deleting Cancellation.}

Let $P$ be an $\mathbb{S L P} \vec{\oplus}$ normalized by the steps Normalization- $\{I, I I\}$. From $P$, we build an SLP $Q$ where $Q$ does not have the XOR cancellation and $\#_{M}(Q) \leq \#_{M}(P)$.

We introduce an auxiliary function unfold to (recursively) unfold the definition of a temporal variable:

$$
\begin{array}{ll}
\operatorname{unfold}(x)=\{x\} & \text { if } x \text { is a constant } \\
\operatorname{unfold}\left(g_{x y}\right)=\left\{g_{x y}\right\} & \\
\operatorname{unfold}(v)=\bigoplus_{i=1}^{n} \operatorname{unfold}\left(t_{i}\right) & \text { if } v \leftarrow t_{1} \oplus t_{2} \oplus \cdots \oplus t_{n}
\end{array}
$$

It should be noted that we do not unfold the definition of a goal for our construction.

Using constants and goals that freely appear in $P$, we define a graph $\supset$ as follows:

(1) First, we define a graph $\supset$ as a complete graph whose nodes are constants that freely appear in $P$.

(2) Next, for each freely appearing goal $g_{x y}$ in $P$, we add the edge $(x, y)$ to $\partial$.

We note the obtained graph $\supset$ may not be connected; i.e., it may have multiple components.

\section{Notations.}

$\partial_{\star}$ : We call the unique component of $\partial$ that contains freely appearing constants $\partial_{\star}$.

$\rightleftharpoons$ : We write $a \rightleftharpoons b$ to the path between $a$ and $b$ of $\partial$.

This graph $\partial$ has the following useful properties.

Proposition A2. Let $v$ be a temporal variable of $P$.

(1) If $\| v \mid=\{x, y\}$, then $x \rightleftharpoons y$.

(2) If $|v|=\left\{x_{1}, x_{2}, x_{3}, x_{4}\right\}$, then this consists of two paths $n_{1} \rightleftharpoons$ $n_{2}$ and $n_{3} \rightleftharpoons n_{4}$ where $\left\{x_{1}, x_{2}, x_{3}, x_{4}\right\}=\left\{n_{1}, n_{2}, n_{3}, n_{4}\right\}$.

(3) If $|v|)=\{\rho, x, y, z\}$, then this consists of one path $n_{1} \rightleftharpoons n_{2}$ and one point $n_{3} \in \partial_{\star}$, where $\{x, y, z\}=\left\{n_{1}, n_{2}, n_{3}\right\}$.

Proof. We show (1). The other cases are shown by the same argument.

Let $X=\operatorname{unfold}(v)$. We reduce $X$ repeatedly applying one of the following replacements rules:

(1) Choose $x \in \partial_{\star}$ and $y \in \partial_{\star}$ from $X$, remove them from $X$, then update $X:=X \oplus\{(x, y)\}$. Remark $(x, y) \in \partial$.
(2) Choose $x \in \partial_{\star}$ and $(x, y) \in \partial$ from $X$, remove them from $X$, then update $X:=X \oplus\{y\}$. Remark $y \in \partial_{\star}$.

(3) Choose $(x, y),(y, z)$ from $X$ where $x \rightleftharpoons y$ and $y \rightleftharpoons z$, remove them them from $X$, then update $X:=X \oplus\{(x, z)\}$. Remark $(x, z) \in$ כ.

When we cannot apply any rule, then $X=\{(a, b)\}$; thus, $a \rightleftharpoons b$.

Proposition A3. If a goal $g_{x y}$ is defined by two temporal variables $v_{1}, v_{2}$ (i.e., $g_{x y} \leftarrow v_{1} \oplus v_{2}$ ), then $x \rightleftharpoons y$.

Proof. By applying the same argument of the above proposition for $X=\operatorname{unfold}\left(v_{1}\right) \oplus \operatorname{unfold}\left(v_{2}\right)$, we have $x \rightleftharpoons y$.

Hereafter, we build an $\operatorname{SLP}_{\vec{\oplus}} Q$ that does not use the XORcancellation

First, we copy $P$ to be normalized to $P^{\prime}$.

Removing nodes that freely appear in $P^{\prime}$. .

Let $a_{1}, a_{2}, \ldots$ are (non-local) nodes that freely appear in $P^{\prime}$,

- If $\Gamma_{a_{i}}$ is in $P^{\prime}$, we move it to $Q$.

- Moving means that we remove $\Gamma_{a_{i}} \leftarrow \rho \oplus a_{i}$ from $P^{\prime}$ and then add it to $Q$.

- Otherwise, by Proposition A1, we have movable occurrences of $\lambda_{a_{i}}$ and $\mu_{a_{i}}$ in $P^{\prime}$.

We remove all the free occurrences of $\Gamma_{a_{i}}$ and all the movable occurrences of $\lambda_{a_{i}}$ and $\mu_{a_{i}}$ from $P^{\prime}$. We then add $\Gamma_{a_{i}} \leftarrow \rho \oplus a_{i}$ to $Q$.

Removing goal variables that freely appear in $P^{\prime}$.

Let $g_{1}, g_{2}, \ldots$ are goal variables that freely appear in $P^{\prime}$.

- Let $g_{i}=g_{a b}$.

- Let $C$ be the component of $\partial$ that the edge $(a, b)$ belongs to.

- If there is $x \in C$ such that $\Gamma_{x}$ is not in $Q$, by Proposition A1, we have movable occurrences of $\lambda_{x}$ and $\mu_{x}$ in $P^{\prime}$.

We remove all the free occurrences of $g_{i}$ and all the movable occurrences of $\lambda_{c}$ and $\mu_{c}$.

We then add $\Gamma_{c} \leftarrow \rho \oplus c$ to $Q$.

After the above modification to $Q$, the following property holds.

Proposition A4.

- $\#_{M}\left(P^{\prime}\right)+\#_{M}(Q) \leq \#_{M}(P)$.

- For each non-local node $a \in \partial_{\star}$, we have $\Gamma_{a}$ in $Q$.

- For each path $a \rightleftharpoons b$, we have $\Gamma_{a}$ or $\Gamma_{b}$ in $Q$.

- For each path $a \rightleftharpoons x$ where $x$ is local, we have $\Gamma_{a}$ in $Q$. We do not need $x \in\left\{\lambda_{a}, \mu_{a}\right\}$.

Removing temporal variables of specific patterns in $P^{\prime}$. Let $v$ be a temporal variable in $P$.

Pat1: If $|v|=\left\{\lambda_{a}, \rho\right\}$ and $\Gamma_{a}$ is not in $Q$, there are movable occurrences of $\lambda_{a}$ and $\mu_{a}$ in $P^{\prime}$.

(1) First, we remove all the such occurrences.

(2) Next, we remove the left-side occurrence of $v$ of the definition of $v$ : i.e.,

$$
v \leftarrow \cdots ; \Leftrightarrow \quad \Leftrightarrow-\leftarrow ;
$$

- Although such the blank is not permitted in our formalization of SLP, we temporarily allow it only on $P^{\prime}$ because we use $P^{\prime}$ to build $Q$ and do not execute $P^{\prime}$. 
- By these removal, we decrement the size of $P^{\prime}$ more than three. Recall that the size of $S L \mathbb{P}_{\vec{\oplus}}$ equals to the number of the total occurrences of constants and variables.

(3) Finally, we add $\Gamma_{a} \leftarrow \rho \oplus a$ to $Q$. It increments the size of $Q$ by three.

Totally, $\#_{M}\left(P^{\prime}\right)+\#_{M}(Q) \leq \#_{M}(P)$ still holds.

Pat2: If $|v|=\left\{\lambda_{a}, \mu_{a}\right\}$ and $\Gamma_{a}$ is not in $Q$, by the same argument of Pat1, we remove the left side occurrence of $v$ and all the movable occurrences of $\lambda_{a}$ and $\mu_{a}$; then, we add $\Gamma_{a} \leftarrow \rho \oplus a$ to $Q$.

Pat3: If $|v|=\left\{\lambda_{a}, b\right\}$ and $\Gamma_{a}$ is not in $Q$, we remove the definition of $v$ from $P^{\prime}$ and then add $\Gamma_{a} \leftarrow \rho \oplus a$ to $Q$.

Pat4: If $|v|=\{a, b\}$ and $\Gamma_{a}$ (or $\Gamma_{b}$ ) is not in $Q$, then we remove the left side occurrence of $v$ and all the movable occurrences of $\lambda_{a}$ and $\mu_{a}$ (or $\lambda_{b}$ and $\mu_{b}$ ) from $P^{\prime}$.

If $\Gamma_{a}$ is not in $Q$, we add $\Gamma_{a} \leftarrow \rho \oplus a$ to $Q$. Otherwise, if $\Gamma_{b}$ is not in $Q$, we add $\Gamma_{b} \leftarrow \rho \oplus b$ to $Q$.

Consequently, we have $\Gamma_{a}$ and $\Gamma_{b}$ in $Q$.

Pat5: If $(v)=\{a, b, c, d\}$, by Proposition A2 and A4 there are at most two nodes $x, y \in\{a, b, c, d\}$ such that $\Gamma_{x}$ and $\Gamma_{y}$ are not in $Q$.

We remove the left side occurrence of $v$ and all the movable occurrences of $\lambda_{x}$, and $\mu_{x}$. We then add $\Gamma_{x} \leftarrow \rho \oplus x$ to $Q$.

Consequently, there is at most one node $y \in\{a, b, c, d\}$ such that $\Gamma_{y}$ is not in $Q$.

Pat6: If $(v)=\left\{a, \lambda_{a}, b, \lambda_{b}\right\}$, by Proposition A2 and A4, $\Gamma_{a}$ or $\Gamma_{b}$ may not exist in $Q$.

If $\Gamma_{a}$ is not in $Q$, we remove the left side occurrence of $v$ and all the movable occurrences of $\lambda_{x}$ and $\mu_{x}$. We then add $\Gamma_{a} \leftarrow \rho \oplus a$ to $Q$.

Otherwise, we do the same for $\Gamma_{b}$. Consequently, we have $\Gamma_{a}$ and $\Gamma_{b}$ in $Q$.

Pat7: If $|v|=\left\{a, \lambda_{a}, b, c\right\}$, by Proposition A2 and A4, we may do not have $\Gamma_{x}$ for at most one of $\{a, b, c\}$.

We remove $v, \lambda_{x}$, and $\mu_{x}$ and then add $\Gamma_{x} \leftarrow \rho \oplus x$.

Consequently, we have $\Gamma_{a}, \Gamma_{b}$, and $\Gamma_{c}$ in $Q$.

Pat8: If $|v|=\left\{\rho, a, \lambda_{a}, b\right\}$, By Proposition A2 and A4, there is at most one node $x \in\{a, b\}$ such that $\Gamma_{x}$ is not in $Q$. For such $x$, we remove the left side occurrence of $v$ and all the movable occurrences of $\lambda_{x}$ and $\mu_{x}$. We then add $\Gamma_{x} \leftarrow \rho \oplus x$ to $Q$.

Consequently, we have $\Gamma_{a}$ and $\Gamma_{b}$ in $Q$.

After the above construction, we still have:

$$
\#_{M}\left(P^{\prime}\right)+\#_{M}(Q) \leq \#_{M}(P) .
$$

Transferring the goal of $P$ without changing its size.

Now we define all the goals without the XOR cancellation in $Q$. First, we consider the goals of the form $g_{a} \lambda_{a}$.

If $g_{a \lambda} \leftarrow p \oplus a \oplus \lambda$ : We move it from $P^{\prime}$ to $Q$.

If $g_{a \lambda} \leftarrow t_{1} \oplus t_{2}$ : Hereafter, for each subcase, we show that we have $\Gamma_{a}$ in $Q$. It suffices for our construction because

(1) We delete the definition of $g_{a \lambda}$; it decrements the size of $P^{\prime}$ by three.

(2) We then add $g_{a \lambda} \leftarrow \Gamma_{a} \oplus \lambda$ to $Q$; it increments the size of $Q$ by three.

(3) Totally, $\#_{M}\left(P^{\prime}\right)+\#_{M}(Q) \leq \#_{M}(P)$ still holds.
Case $g_{a \lambda} \leftarrow p \oplus a\left(\lambda\right.$ has been removed to add $\Gamma_{a}$ to $\left.Q\right)$ : Clearly we have $\Gamma_{a}$ in $Q$.

Case $g_{a \lambda} \leftarrow v \oplus a$ and $|v|=\{\lambda, \rho\}$ : By Pat1, we have $\Gamma_{a}$ in $Q$.

Case $g_{a \lambda} \leftarrow \Gamma_{a} \oplus \lambda$ : Clearly we have $\Gamma_{a}$ in $Q$.

Case $g_{a \lambda} \leftarrow v \oplus \rho$ and $(v)=\left\{a, \lambda_{a}\right\}$ : By Proposition A2 and A4, we have $\Gamma_{a}$ in $Q$.

Case $g_{a \lambda} \leftarrow v \oplus b$ and $|v|=\left\{\rho, a, \lambda_{a}, b\right\}$ : By Pat8, we have $\Gamma_{a}$ and $\Gamma_{b}$ in $Q$

Case $g_{a \lambda_{a}} \leftarrow v \oplus g_{a \mu_{a}}$ and $(v)=\left\{\lambda_{a}, \mu_{a}\right\}$; By Pat2, we have $\Gamma_{a}$ in $Q$.

Case $g_{a \lambda_{a}} \leftarrow v \oplus g_{a b}$ and $(v)=\left\{\lambda_{a}, b\right\}$ : By Pat3, we have $\Gamma_{a}$ in $Q$.

Case $g_{a \lambda_{a}} \leftarrow v \oplus g_{b \lambda_{b}}$ and $(v)=\left\{a, \lambda_{a}, b, \lambda_{b}\right\}$ : By Pat6, we have $\Gamma_{a}$ in $Q$;

Case $g_{a \lambda_{a}} \leftarrow v \oplus g_{b c}$ and $(v)=\left\{a, \lambda_{a}, b, c\right\}$ : By Pa7, we have $\Gamma_{a}$ in $Q$

Case $g_{a \lambda_{a}} \leftarrow v_{1} \oplus v_{2}$ and $v_{1}, v_{2}$ are variables: By Proposition A3 and $\mathrm{A} 4$, we have $\Gamma_{a}$ in $Q$.

Next, we consider the goals of the form $g_{a b}$ where $a$ and $b$ are (non-local) nodes.

If $g_{a b} \leftarrow \rho \oplus a \oplus b$ : We copy it to $Q$.

If $g_{a b} \leftarrow t_{1} \oplus t_{2}$ : Hereafter, for each subcase, we show that we have $\Gamma_{a}$ in $Q$. As the same as the construction of $g_{a} \lambda_{a}$, it suffices for out construction.

If $g_{a b} \leftarrow \Gamma_{a} \oplus b$ : Clearly, we have $\Gamma_{a}$ in $Q$.

If $g_{a b} \leftarrow v \oplus \rho$ and $(v)=\{a, b\}$ : By Proposition A2 and A4, we have $\Gamma_{a}$ or $\Gamma_{b}$ in $Q$.

If $g_{a b} \leftarrow v \oplus \lambda_{a}$ and $(v)=\left\{\rho, a, b, \lambda_{a}\right\}$ : By Proposition A2 and A4, we have $\Gamma_{a}$ or $\Gamma_{b}$ in $Q$.

If $g_{a b} \leftarrow v \oplus c$ and $(v)=\{\rho, a, b, c\}$ : By Proposition A2 and A4, we have $\Gamma_{a}$ or $\Gamma_{b}$ in $Q$.

If $g_{a b} \leftarrow v \oplus g_{a \lambda_{a}}$ and $|v|=\left\{b, \lambda_{a}\right\}$ : By Pat3, we have $\Gamma_{a}$ in $Q$.

If $g_{a b} \leftarrow v \oplus g_{a c}$ and $|v|=\{b, c\}:$ By Pat4, we have $\Gamma_{b}$ and $\Gamma_{c}$ in $Q$.

If $g_{a b} \leftarrow v \oplus g_{c \lambda_{c}}$ and $\left.\| v\right)=\left\{a, b, c, \lambda_{c}\right\}$ : By Pat7, we have $\Gamma_{a}$ or $\Gamma_{b}$ in $Q$.

If $g_{a b} \leftarrow v \oplus g_{c d}$ and $\left.\| v\right\rangle=\{a, b, c, d\}$ : By Pat5, we have $\Gamma_{a}$ or $\Gamma_{b}$ in $Q$.

If $g_{a b} \leftarrow v_{1} \oplus v_{2}$ : By Proposition A3 and A4, we have $\Gamma_{a}$ or $\Gamma_{b}$ in $Q$.

By our construction, the following holds in $Q$.

LEMma A4.

- $\llbracket Q \rrbracket=\llbracket P \rrbracket$.

- $\#_{M}(Q) \leq \#_{M}(P)$.

- Every temporal variable of $Q$ is the form of $\Gamma_{a} \leftarrow \rho \oplus a$ where a is a node of $G$.

- The definition of each goal $g$ of $Q$ forms one of the following: $-g \leftarrow \Gamma_{a} \oplus x$

$-g \leftarrow \rho \oplus x \oplus y$

A.3.4 Normalization IV: Finalization.

Finally, we prove Lemma A1. 
The above lemma Lemma A4 does not ensure that every edge $(a, b)$ is represented by $g_{a b} \leftarrow \Gamma_{a} \oplus b$ or $g_{a b} \leftarrow \Gamma_{b} \oplus a$ because $Q$ may not have both of $\Gamma_{a}$ and $\Gamma_{b}$.

In the case, $Q$ has the following definitions:

$$
\begin{aligned}
& g_{a b} \leftarrow \rho \oplus a \oplus b ; \\
& g_{a \lambda} \leftarrow \rho \oplus a \oplus \lambda ; \\
& g_{a \mu} \leftarrow \rho \oplus a \oplus \mu ;
\end{aligned}
$$

We change this part as follows without changing $\#_{M}(Q)$ :

$$
\begin{aligned}
& \Gamma_{a} \leftarrow \rho \oplus a ; \\
& g_{a b} \leftarrow \Gamma_{a} \oplus b ; \\
& g_{a \lambda} \leftarrow \Gamma_{a} \oplus \lambda ; \\
& g_{a \mu} \leftarrow \Gamma_{a} \oplus \mu ;
\end{aligned}
$$

Repeatedly applying this modification, we transform $Q$ to $Q^{\prime}$ that satisfies all the conditions of Lemma A1. 